\title{
THE FARTHEST KNOWN SUPERNOVA: SUPPORT FOR AN ACCELERATING UNIVERSE AND A GLIMPSE OF THE EPOCH OF DECELERATION ${ }^{1}$
}

\author{
Adam G. Riess, ${ }^{2}$ Peter E. Nugent, ${ }^{3}$ Ronald L. Gilliland, ${ }^{2}$ Brian P. Schmidt, ${ }^{4}$ John Tonry, ${ }^{5}$ Mark Dickinson, ${ }^{2}$ \\ Rodger I. Thompson, ${ }^{6}$ Tamás Budavári, ${ }^{7}$ Stefano Casertano, ${ }^{2}$ Aaron S. Evans, ${ }^{8}$ Alexei V. Filippenko, ${ }^{9}$ \\ Mario Livio, ${ }^{2}$ David B. Sanders, ${ }^{5}$ Alice E. Shapley, ${ }^{10}$ Hyron Spinrad, ${ }^{9}$ Charles C. Steidel, ${ }^{10}$ \\ Daniel STERn, ${ }^{11}$ Jason Surace, ${ }^{12}$ AND SYlVAin VeIlleux ${ }^{13}$ \\ Received 2001 March 12; accepted 2001 May 18
}

\begin{abstract}
We present photometric observations of an apparent Type Ia supernova ( $\mathrm{SN}$ Ia) at a redshift of $\sim 1.7$, the farthest SN observed to date. The supernova, SN 1997ff, was discovered in a repeat observation by the Hubble Space Telescope (HST) of the Hubble Deep Field-North (HDF-N) and serendipitously monitored with NICMOS on HST throughout the Thompson et al. Guaranteed-Time Observer (GTO) campaign. The SN type can be determined from the host galaxy type: an evolved, red elliptical lacking enough recent star formation to provide a significant population of core-collapse supernovae. The classification is further supported by diagnostics available from the observed colors and temporal behavior of the SN, both of which match a typical SN Ia. The photometric record of the SN includes a dozen flux measurements in the $I, J$, and $H$ bands spanning 35 days in the observed frame. The redshift derived from the SN photometry, $z=1.7 \pm 0.1$, is in excellent agreement with the redshift estimate of $z=1.65 \pm 0.15$ derived from the $U_{300} B_{450} V_{606} I_{814} J_{110} J_{125} H_{160} H_{165} K_{s}$ photometry of the galaxy. Optical and near-infrared spectra of the host provide a very tentative spectroscopic redshift of 1.755 . Fits to observations of the SN provide constraints for the redshift-distance relation of $\mathrm{SNe} \mathrm{Ia}$ and a powerful test of the current accelerating universe hypothesis. The apparent SN brightness is consistent with that expected in the decelerating phase of the preferred cosmological model, $\Omega_{M} \approx 1 / 3, \Omega_{\Lambda} \approx \frac{2}{3}$. It is inconsistent with gray dust or simple luminosity evolution, candidate astrophysical effects that could mimic previous evidence for an accelerating universe from $\mathrm{SNe}$ Ia at $z \approx 0.5$. We consider several sources of potential systematic error, including gravitational lensing, supernova misclassification, sample selection bias, and luminosity calibration errors. Currently, none of these effects alone appears likely to challenge our conclusions. Additional SNe Ia at $z>1$ will be required to test more exotic alternatives to the accelerating universe hypothesis and to probe the nature of dark energy.
\end{abstract}

Subject headings: cosmology: observations - supernovae: general

On-line material: color figure

\footnotetext{
${ }^{1}$ Based on observations with the NASA/ESA Hubble Space Telescope, obtained at the Space Telescope Science Institute, which is operated by AURA, Inc., under NASA contract NAS 5-26555.

${ }^{2}$ Space Telescope Science Institute, 3700 San Martin Drive, Baltimore, MD 21218; ariess@stsci.edu.

${ }^{3}$ Lawrence Berkeley National Laboratory, Berkeley, CA 94720.

${ }^{4}$ Mount Stromlo and Siding Spring Observatories, Private Bag, Weston Creek P.O. 2611, Australia.

5 Institute for Astronomy, University of Hawaii, 2680 Woodlawn Drive, Honolulu, HI 96822

${ }^{6}$ Steward Observatory, University of Arizona, Tucson, AZ 85721.

${ }^{7}$ Department of Physics and Astronomy, The Johns Hopkins University, Baltimore, MD 21218, and Department of Physics, Eötvös University, Budapest, Pf. 32, Hungary, H-1518.

${ }^{8}$ Department of Physics and Astronomy, State University of New York (SUNY) at Stony Brook, NY 11794-3800.

${ }^{9}$ Department of Astronomy, University of California, Berkeley, CA 94720-3411.

${ }^{10}$ Palomar Observatory, California Institute of Technology, Mail Code 105-24, Pasadena, CA 91125.

11 Jet Propulsion Laboratory, California Institute of Technology, Mail Code 169-327, Pasadena, CA 91109.

${ }^{12}$ SIRTF Science Center, California Institute of Technology, Mail Code 314-6, Pasadena, CA 91125.

${ }^{13}$ Department of Astronomy, University of Maryland, College Park, MD 20742.
}

\section{INTRODUCTION}

The unexpected faintness of Type Ia supernovae ( $\mathrm{SNe} \mathrm{Ia})$ at $z \approx 0.5$ provides the most direct evidence that the expansion of the universe is accelerating, propelled by "dark energy" (Riess et al. 1998; Perlmutter et al. 1999). This conclusion is supported by measurements of the characteristic angular scale of fluctuations in the cosmic microwave background (CMB) that reveal a total energy density well in excess of the fraction attributed to gravitating mass (de Bernardis et al. 2000; Balbi et al. 2000; Jaffe et al. 2001).

However, contaminating astrophysical effects can imitate the evidence for an accelerating universe. A pervasive screen of gray dust could dim SNe Ia with little telltale reddening apparent from their observed colors (Aguirre 1999a, 1999b; Rana 1979, 1980). Although the first exploration of a distant SN Ia at near-infrared (NIR) wavelengths provided no evidence of nearly gray dust, more data are needed to perform a definitive test (Riess et al. 2000).

A more familiar challenge to the measurement of the global acceleration or deceleration rate is luminosity evolution (Sandage \& Hardy 1973). The lack of a complete theo- 
retical understanding of SNe Ia and an inability to identify their specific progenitor systems undermines our ability to predict with confidence the direction or degree of luminosity evolution (Höflich, Wheeler, \& Thielemann 1998; Umeda et al. 1999a, 1999b; Livio 2000; Drell, Loredo, \& Wasserman 2000; Pinto \& Eastman 2000; Yungelson \& Livio 2000). The weight of empirical evidence appears to disfavor evolution as an alternative to dark energy as the cause of the apparent faintness of SNe Ia at $z \approx 0.5$ (see Riess 2000 for a review). However, the case against evolution remains short of compelling.

The extraordinary claim of the existence of dark energy requires a high level of evidence for its acceptance. Fortunately, a direct and definitive test is available. It should be possible to discriminate between cosmological models and "impostors" by tracing the redshift-distance relation to redshifts greater than one.

\subsection{The Next Redshift Octave and the Epoch of Deceleration}

If the cosmological acceleration inferred from $\mathrm{SNe}$ Ia is real, it commenced rather recently, at $0.5<z<1$. Beyond these redshifts, the universe was more compact and the attraction of matter dominated the repulsion of dark energy. At $z>1$, the expansion of the universe should have been decelerating (see Filippenko \& Riess 2000). The observable result at $z \geq 1$ would be an apparent increased brightness of SNe Ia relative to what is expected for a nondecelerating universe. However, if the apparent faintness of $\mathrm{SNe}$ Ia at $z \approx 0.5$ is caused by dust or simple evolution, $\mathrm{SNe}$ Ia at $z>1$ should appear fainter than expected from decelerating cosmological models. More complex parameterizations of evolution or extinction that can match both the accelerating and decelerating epochs of expansion would require a higher order of fine-tuning and are therefore less plausible.

Measuring global deceleration at $z>1$ provides additional cosmological benefits. To constrain the equation of state of dark energy (and distinguish a cosmological constant from the decaying scalar fields described by the "quintessence" hypothesis; Peebles \& Ratra 1988; Caldwell, Davé, \& Steinhardt 1998), it is necessary to break degeneracies that exist between the global densities of mass and dark energy. Observations of SNe Ia in this next redshift octave are well suited to deciphering the nature of dark energy and have motivated recent proposals to develop a wide-field optical and NIR space mission (Curtis et al. $2000^{14}$; Nugent 2000). To better determine the merits and technical requirements of such a mission, it will be important to study closely the first few $\mathrm{SNe}$ detected at these redshifts. In addition, the study of $\mathrm{SNe}$ Ia at $z>1$ can provide meaningful constraints on progenitor models (Livio 2000; Nomoto et al. 2000; Ruiz-Lapuente \& Canal 1998) after surveys of such $\mathrm{SNe}$ over a range of high redshifts are completed.

Both the Supernova Cosmology Project (SCP; Perlmutter et al. 1995) and the High-z Supernova Search Team (HZT; Schmidt et al. 1998) have pursued the discovery of $\mathrm{SNe}$ Ia in this next redshift interval. In the fall of 1998, the SCP reported the discovery of SN 1998ef at $z=1.2$ (Aldering et al. 1998). The following year, the HZT dis-

\footnotetext{
${ }^{14}$ See D. Curtis et al. 2000, Supernova/Acceleration Probe (SNAP), proposal to DOE and NSF at http://snap.lbl.gov/.
}

covered an SN Ia at $z=1.2$ (SN 1999fv) as well as at least one more at $z \approx 1.05$ (Tonry et al. 1999; Coil et al. 2000). These data sets, while currently lacking the statistical power to discriminate between cosmological and astrophysical effects, are growing and may provide the means to break degeneracies in the future.

In early 1998, Gilliland \& Phillips (1998) reported the detection of two SNe, SN 1997ff and SN 1997fg, in a reobservation of the Hubble Deep Field-North (HDF-N) with WFPC2 through the F814W filter. The elliptical host of SN $1997 \mathrm{ff}$ indicated that this supernova was "most probably a SN Ia...[at] the greatest distance reported previously for SNe," but the observations at a single epoch and in a single band were insufficient to provide useful constraints on the SN and, hence, to perform cosmological tests (Gilliland, Nugent, \& Phillips 1999, hereafter GNP99).

Here we report additional, serendipitous observations of SN 1997ff obtained in the Guaranteed-Time Observer (GTO) NICMOS campaign (Thompson et al. 1999) and in the General Observer (GO) program 7817 (M. Dickinson et al. 2001, in preparation), as well as spectroscopy of the host. The combined data set provides the ability to put strong constraints on the redshift and distance of this supernova and shows it to be the highest redshift SN Ia observed (to date). These measurements further provide an opportunity to perform a new and powerful test of the accelerating universe by probing its preceding epoch of deceleration.

In $\S 2$ of this paper, we describe the observations of the $\mathrm{SN}$ and its host in the HDF-N and report photometry of the $\mathrm{SN}$ from the NICMOS campaign. In $\S 3$, we analyze the observations to constrain the SN parameters: redshift, luminosity, age of discovery, and distance. The constraints are used to extend the distance-redshift relation of SNe Ia to $z>1$ and to discriminate between cosmological models and contaminating astrophysical effects. Section 4 contains a discussion of the systematic uncertainties in our measurements and their implications. We summarize our findings in $\S 5$.

\section{OBSERVATIONS}

\subsection{The Discovery of $S N 1997 f f$}

Between 1997 December 23 and December 26, Gilliland \& Phillips (GO 6473) reobserved the HDF-N with the Hubble Space Telescope (HST) to detect high-redshift SNe. These observations were obtained with WFPC2 (F814W) during $18 H S T$ orbits in the continuous viewing zone (CVZ) and at a spacecraft orientation as closely matched to the original HDF-N as possible. To critically sample the WF point-spread function (PSF) and robustly reject all hot pixels, CCD defects, and noise fluctuations, the exposures were well dithered using 18 different subpixel and multipixel offsets. Additional F300W frames were obtained during the bright portion of the CVZ orbits to support improved rejection of transient hot pixels. The total F814W exposure time in the second HDF-N epoch (6300 s) was $51 \%$ of that obtained in the original epoch $2.0 \mathrm{yr}$ prior.

After careful processing, the second epoch was registered with the first and difference frames in both temporal directions were produced. Robust SN detection thresholds were determined by a Monte Carlo exercise of adding PSFs of varying brightness onto host galaxies of varying redshifts. From this exercise, it was determined that a brightness threshold of $m_{I}<27.7$ (Johnson-Cousins) would ensure the 
rejection of all spurious transients. Simulations of completeness indicated that $95 \%$ of SNe at $m_{I}=27$ coincident with host galaxies at $1.5<z<1.9$ would be discovered. Only transients that were brighter than the rejection threshold, visible in each of three subsets and near a host galaxy, were identified as SNe. Candidates coincident with hosts' centers were discarded as possible active galactic nuclei. The harvest from the HDF-N SN search was two robust SN detections: SN 1997fg and SN 1997ff at a signal-to-noise ratio $(\mathrm{S} / \mathrm{N})$ of 20 and 9 , respectively. The former was hosted by a late-type galaxy with a spectroscopic redshift of 0.95 .

SN $1997 \mathrm{ff}$ was discovered at $m_{I}=27.0 \mathrm{mag}$, R.A. $=$ $12^{\mathrm{h}} 36^{\mathrm{m}} 44.11$, decl. $=+62^{\circ} 12^{\prime} 44^{\prime \prime} .8$ (equinox J2000), $0^{\prime \prime} .16$ southwest of the center of the host galaxy, 4-403.0 (Williams et al. 1996). The host has been classified as an elliptical galaxy based on measurements of its surface brightness profile, concentration, asymmetry, and colors as well as by visual inspection (Williams et al. 1996; Fernandez-Soto, Lanzetta, \& Yahil 1999; Dickinson 1999; Thompson et al. 1999; Budavári et al. 2000). A section of the HDF-N near the SN host is shown in Figure 1 as observed with WFPC2 and NICMOS. GNP99 favored the classification of SN $1997 \mathrm{ff}$ as Type Ia because of the red, elliptical host.

Photometric redshift determinations of the host had been published using only $U_{300} B_{450} V_{606} I_{814}$ photometry of the HDF-N ( $z=0.95$; Sawicki, Lin, \& Yee 1997), as well as from the later addition of $J_{125} H_{165} K_{s}$ data from the ground ( $z=1.32$; Fernandez-Soto et al. 1999). To span the rest-frame optical breaks in the spectral energy distribution (SED) of galaxies with $z>1$ and reliably estimate their photometric redshift, it is necessary to employ both optical and NIR data. GNP99 assumed the Fernandez-Soto et al. (1999) redshift which employed the best available coverage of the host SED to date. However, even with the monochromatic detection of a probable SN Ia and an estimate of its redshift, the extraction of useful cosmological information from SN 1997ff was not feasible and was not attempted.

\subsection{Serendipity: The NICMOS Campaigns}

Two NIR assaults on the HDF-N with NICMOS on $H S T$ provided a wealth of data and understanding on the natural history of galaxies (see Ferguson, Dickinson, \& Williams 2000 for a review). The GTO program of Thompson et al. (1999; GTO 7235) consisted of $\sim 100$ orbits of F110W and F160W exposures of a single 55" $\times 55^{\prime \prime}$ Camera-3 field, reaching a limiting AB magnitude (Oke \& Gunn 1983) of 29 in the latter. The observations were gathered during 14 consecutive days and the field was contained within the WF4 portion of HDF-N, serendipitously imaging the host of SN $1997 \mathrm{ff}$. (It is interesting to note that the placement of the GTO field within the HDF-N had less than a $20 \%$ chance of containing SN 1997ff.) Although the program did not begin until 1998 January 19, about 25 days after the discovery of the $\mathrm{SN}$, a series of single-dither exposures (GO 7807) was taken between the discovery of the $\mathrm{SN}$ and the start of the GTO program for the purpose of verifying the suitability of the chosen guide stars. Each of these exposures was for a duration of 960 s. A single F110W and F240M exposure on 1998 January 6 included the host, as did a F160W exposure from 1998 January 2 and another on 1997 December 26. The December 26 NICMOS exposure was coincident within hours of the WFPC2 discovery exposures. (It is of further interest to note the low likelihood of the chance temporal coincidence of the HDF-N SN Search and the GTO program, each initially scheduled in different $H S T$ cycles.)

A second program was undertaken 6 months after the GTO program, between 1998 June 14 and June 22, by M. Dickinson et al. (2001, in preparation; GO 7817). This program observed the entire HDF-N in F110W and F160W to a limiting AB magnitude of $\sim 26.5$ by mosaicing Camera 3 of NICMOS to study a wider field of galaxies. This program also contained the host galaxy and the greatly faded light of the supernova. The space-based NIR photometry of the SN host offered greater precision and coverage of the SED than the ground-based data alone and allowed an improved estimate of the photometric redshift. Using the space-based $U_{300} B_{450} V_{606} I_{814} J_{110} H_{160}$ photometry and the ground-based $J_{125} H_{165} K_{s}$ photometry contained in Table 1, Budavári et al. (2000) determined the redshift of the host to be $z=1.65 \pm 0.15$ from fits to either galaxy SED eigenspectra or these same eigenspectra mildly
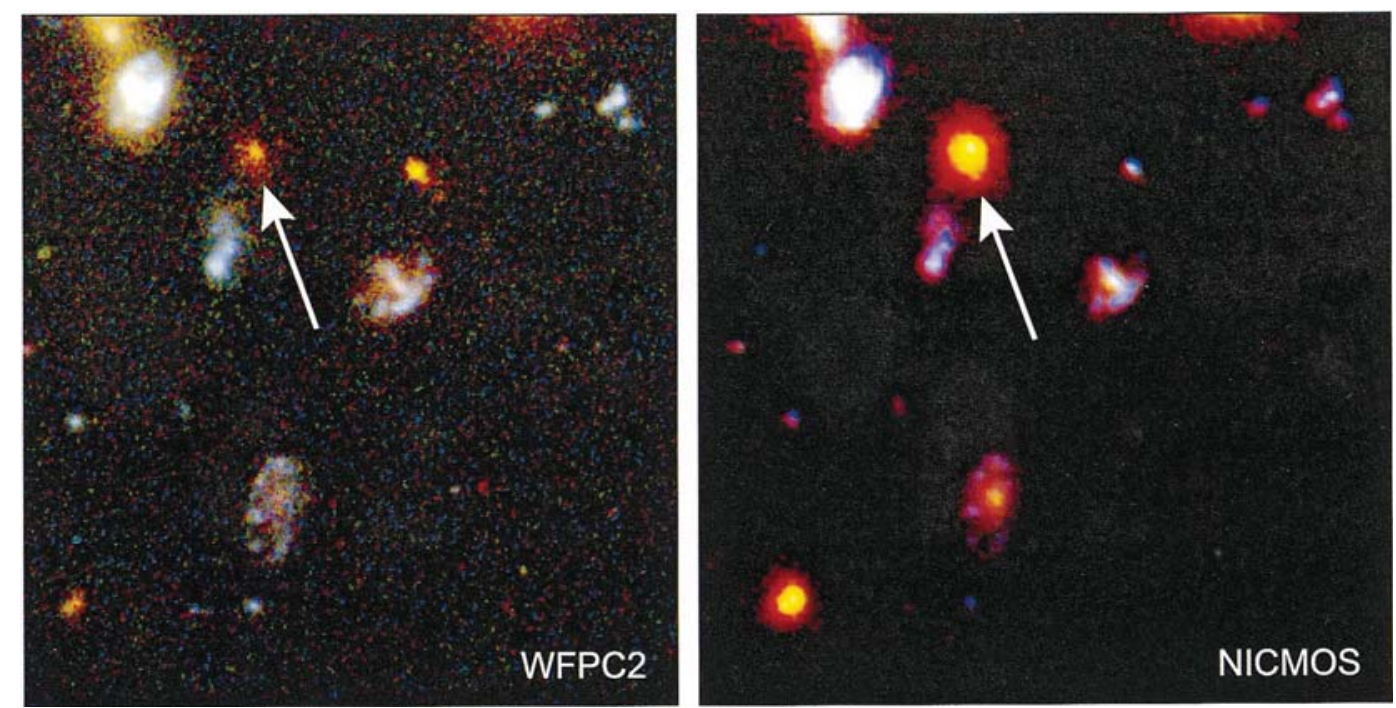

FIG. 1.-Color-composite images of the region of the HDF-N near the host of SN 1997ff. The WFPC2 images were taken during the HDF-N campaign (Williams et al. 1996) and the NICMOS images were taken during the GTO campaign (Thompson et al. 1999). The arrow indicates the SN host galaxy. 
TABLE 1

AB Magnitudes of the Host GalaXY oF SN 1997FF

\begin{tabular}{|c|c|c|}
\hline Bandpass & $\log (\lambda / \mu \mathrm{m})$ & AB Magnitude \\
\hline$F U V_{160}{ }^{\mathrm{b}}$. & -0.80 & $<30.0$ \\
\hline$N U V_{250}{ }^{\mathrm{b}} \ldots \ldots$ & -0.60 & $<29.2$ \\
\hline$U_{300} \ldots \ldots \ldots$ & -0.53 & $27.84_{-0.70}^{+2.48}$ \\
\hline$B_{450} \ldots \ldots \ldots \ldots$ & -0.34 & $26.67 \pm 0.16$ \\
\hline$V_{606} \ldots \ldots \ldots \ldots$ & -0.22 & $25.64 \pm 0.04$ \\
\hline$I_{814} \ldots \ldots \ldots \ldots$ & -0.10 & $24.42 \pm 0.02$ \\
\hline$J_{110} \ldots \ldots \ldots \ldots$ & 0.04 & $22.60 \pm 0.02$ \\
\hline$J_{125}{ }^{\mathrm{a}} \ldots \ldots \ldots \ldots$ & 0.10 & $21.96 \pm 0.03$ \\
\hline$H_{160} \ldots \ldots \ldots \ldots$ & 0.20 & $21.59 \pm 0.01$ \\
\hline$H_{165}{ }^{\mathrm{a}} \ldots \ldots \ldots$ & 0.22 & $21.55 \pm 0.03$ \\
\hline$K_{s}^{\mathrm{a}} \ldots \ldots \ldots \ldots$ & 0.33 & $21.03 \pm 0.02$ \\
\hline
\end{tabular}

corrected to improve the agreement between spectroscopic and photometric redshifts. The redshift constraints derived from the host photometry are analyzed in $\S 3.1$.

\subsection{Supernovae Photometry}

The two HDF-N NICMOS campaigns taken together offer a rare opportunity to measure the behavior of a supernova at a redshift not accessible from the ground and perhaps to discriminate between the influence of dark energy and contaminating astrophysical effects at $z>1$. For favored cosmological models $\left(\Omega_{M} \approx \frac{1}{3}, \Omega_{\Lambda} \approx \frac{2}{3}\right)$, an SN Ia at $z=1.65$ is expected to peak in F160W at $\sim 24$ mag and in F110W at $\sim 24.5 \mathrm{mag}$. The $130 \mathrm{ks}$ of exposure time in each bandpass of the GTO program would be expected to reach $\mathrm{S} / \mathrm{N} \approx 100$ for an $\mathrm{SN}$ Ia at peak, though actual measurements impacted by the shot noise of the bright host may be more uncertain. Because the $\mathrm{SN}$ would not be at peak for some or all of the observations, we expect further reductions in the measurement precision. If the apparent brightnesses of high-redshift $\mathrm{SNe}$ are dominated by evolution and/or dust, and not by cosmology, the $\mathrm{S} / \mathrm{N}$ of the $\mathrm{SN}$ might be further reduced. For the single dithers used to test guide stars, we expect an $\mathrm{S} / \mathrm{N}$ no better than $\sim 10$ and possibly worse because of the above mitigating factors.

Another valuable and fortuitous feature of the NICMOS campaigns is that they likely sample the rest-frame light of the supernova in the $B$ and $V$ bands, the most studied and best understood wavelength region of nearby $\mathrm{SNe}$. Indeed, the great difficulty in observing these wavelengths from the ground has generally limited the detection and monitoring of SNe Ia to $z \lesssim 1$. In the $\sim 2$ rest-frame months expected to have elapsed between the two separate NICMOS programs, an SN Ia is expected to fade $\sim 3 \mathrm{mag}$, resulting in a significant surplus of flux in the difference image of the two epochs.

Our goal is to measure the photometry of the $\mathrm{SN}$ throughout the 35 days of the GTO program. However, our task is complicated by the proximity of the SN to its bright host. GNP99 found that the SN was located at the half-light radius of the galaxy in $\mathrm{F} 814 \mathrm{~W}$. As we will find, the host contains 2 to 6 times as much flux at the position of the SN as the peak of the SN PSF, depending on the band and the date of the exposure. The strategy of digitally subtracting an image of the host obtained when the SN has faded (template image) from one taken when the $\mathrm{SN}$ is relatively bright has been successfully employed by the SCP (Perlmutter et al. 1995 ) and the High-z Team (Schmidt et al. 1998), as well as by GNP99 using the original HDF-N images as a template image. This is the method we employed.

The task of geometrically mapping (i.e., registering) the images from the Thompson et al. campaign to align with the template image from the Dickinson et al. campaign was complicated by the timing and field location of the former. As seen in Figure 2, the exposure time for the SN in the Thompson et al. campaign was dispersed irregularly over a 35 day time interval, requiring careful consideration of the optimal way to measure the temporal behavior of the SN while still yielding robust photometry (see below). In addition, the location of the SN was always extremely close to the corner of the Camera- 3 field of $256 \times 256$ pixels, missing the chip during one-third of the dithers, and landing 1 to 15 pixels from the corner in the rest. Although the Camera-3 field is remarkably distortion-free in its interior, a mild degree of "pincushion" distortion exists in the extreme corners. Even distortions of a few tenths of a pixel are intolerable for the accurate subtraction of the host flux from the SN (Cox et al. 1997). Although application of the NIC-3 geometric distortion map removes much of the distortion in the field corners, we applied an empirically derived linear mapping between the SN image and template to further reduce host contamination.

Our first step was to use the SExtractor algorithm (Bertin \& Arnouts 1996) to detect sources and measure their centroids in the template and SN images. Custom software was used to match identical sources in the two lists (Schmidt et al. 1998). Next, the geomap routine in IRAF was used to derive a flux-conserving mapping of the $\mathrm{SN}$ images to the template coordinate system. In practice we found that many of the individual $900 \mathrm{~s}$ dithers in the GTO campaign did not provide the desired $\mathrm{S} / \mathrm{N}$ in the centroid measurements to

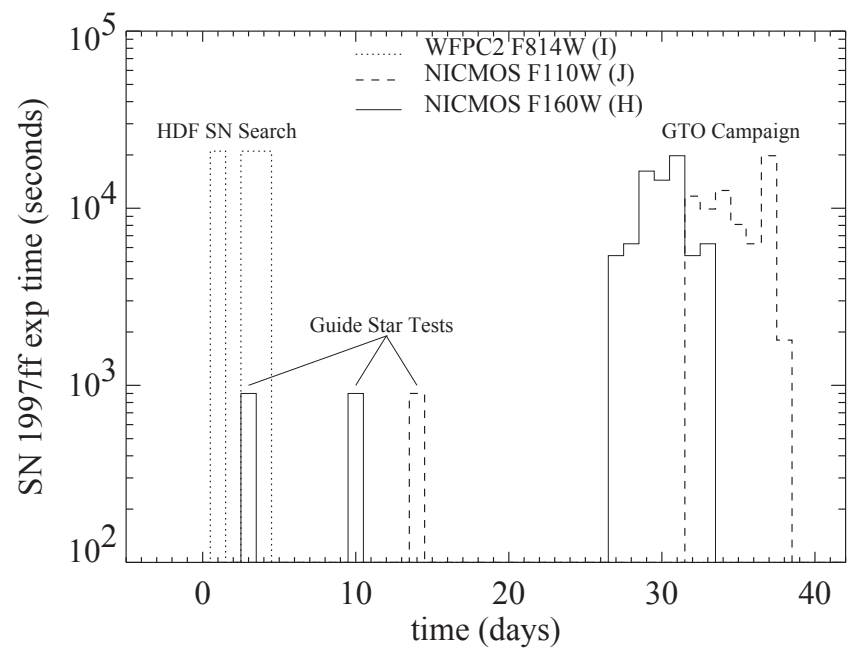

FIG. 2.- HST exposures obtained for SN 1997ff as a function of time in different bandpasses. Time in days (the abscissa) is given relative to 1997 December 23, the start of the HDF-N SN search with WFPC2 (GO 6473). The subsequent GTO campaign with NICMOS (Thompson et al. 1999; GTO 7235) and its preceding tests for guide star suitability (7807) provided valuable coverage of the light curve of the $\mathrm{SN}$ found during the search. A subsequent NICMOS campaign (M. Dickinson et al. 2001, in preparation; GO 7817) provided templates to remove the contaminating light of the host after the SN had faded. 
derive a robust mapping to the template coordinate system. A tenable alternative is to first drizzle together a subset of the dithers (Fruchter \& Hook 1997; Thompson et al. 1999; GNP99) before deriving the nonlinear coordinate transform. This practice has the advantage of increasing the precision of the centroid measurements of the sources, allowing for the critical sampling of the PSF, reducing the effects of NICMOS interpixel sensitivity (Storrs et al. 1999), and providing the ability to further remove cosmic rays (Thompson et al. 1999). However, the obvious disadvantage of combining the dithers before further processing (i.e., binning) is a reduction in our ability to resolve the temporal behavior of the SN. After much experimentation, we chose an intermediate strategy of combining the dithers from the main GTO campaign into three temporal bins of observed-frame width $\sim 2$ days for each of the F110W and F160W data sets. A similar strategy was used by GNP99 to provide time-resolved magnitudes of the $\mathrm{SN}$ in F814W. The necessary exception to the practice of binning was for the measurement of the SN flux in the individual $960 \mathrm{~s}$ dithers used to test guide stars before the GTO campaign.

We employed the Alard (2000) algorithm to match the PSF, mean intensity, and background in the template and SN images. Using the nearest visible sources to the SN's position, we first derived and then applied a convolution kernel with a linear variation across the sources. (Again, experimentation showed a constant convolution kernel was inadequate for matching the two image PSFs, and secondorder convolutions were unstable because of a lack of enough sources with sufficient $\mathrm{S} / \mathrm{N}$ to measure the kernel variation.) Next, we subtracted the template image from the SN images. As seen in Figure 3, the resulting image contains the SN without the contaminating light of the host. An important test of these image-processing routines is to verify a lack of significant flux residuals in the vicinity of other galaxies (which did not host $\mathrm{SNe}$ ) in the field. Confirmation of this test can be seen in Figure 3 (see also GNP99, Fig. 1 for the comparable F814W discovery images of SN 1997ff).

Next, we measured the flux of the SN. Again, the format of the NICMOS campaigns (optimized for galaxy studies, not for the monitoring of SN 1997ff) presented challenges rarely encountered by past high-redshift supernova programs. Typically, the leading source of noise in the measurements of the flux in high-redshift $\mathrm{SNe}$ is the shot noise in the sky (or the host galaxy in HST observations). For the NICMOS observations of SN 1997ff, the dominant source of uncertainty is the host galaxy residuals in the difference image. The location of the SN near the core of a much brighter host results in the appearance of significant galaxy residuals from typically tolerable errors of 0.1 pixels in the image pair registration. Eradicating registration errors at the position of the $\mathrm{SN}$ is made more difficult by the location of the host galaxy near the corner of the Camera-3 field in the Thompson et al. (1999) campaign. For some images, we reduced this error by processing only a subset of the image around the SN. Galaxy residuals in the difference image can also result from the undersampling of Camera 3 and from variations of the intrapixel sensitivity. To derive a robust measure of the SN flux, we used a relatively large aperture to contain the net flux near the $\mathrm{SN}$ impacted by the galaxy residuals. In practice, apertures with a radius of 5 to 10 oversampled-by-two pixels $\left(0.5\right.$ to $\left.1^{\prime \prime} .0\right)$ were used. The flux of the SN was measured relative to that contained in an equal-sized aperture of a bright comparison star at the center of the GTO field. Flux uncertainties were determined by a Monte Carlo exercise of adding and measuring artificial $\mathrm{SNe}$ in the field with the same brightness and background as SN 1997ff (Schmidt et al. 1998). For the single F110W dither from 1998 January 6, the SN was not detected, but a flux upper limit was established by the Monte Carlo exercise.

We transformed the relative $\mathrm{SN}$ flux onto the $\mathrm{F} 110 \mathrm{~W} \mathrm{AB}$ and $\mathrm{F} 160 \mathrm{~W}$ AB magnitude systems by applying the zero points of the transformation equation from M. Dickinson et al. (2001, in preparation). A count rate for the comparison star of 3.22 and 1.85 ADU per second was measured in F110W and F160W, respectively. Using the zero points of 22.89 for AB F110W and 22.85 for AB F160W gave a measured magnitude of 21.62 and 22.18 for the star in these two bandpasses, respectively. (An expected uncertainty of $\sim 5 \%$ in these zero points is insignificant in comparison with the uncertainties in the relative photometry.) Addition of the measured magnitude differences between the star and the $\mathrm{SN}$ yielded the measurements for the $\mathrm{SN}$ on the AB system. To transform the $\mathrm{AB}$ system magnitudes onto the Vega system, we calculated the bandpass-weighted magnitudes of spectrophotometry of Vega (relative to a flat spectrum) and derived the zero-point offsets of -1.34 and $-0.75 \mathrm{mag}$ for F160W and F110W, respectively. The Vega system F160W and F110W magnitudes are given in Table 2, as are the F814W Vega system magnitudes from GNP99. It is important to note that the magnitudes of the SN in F110W and F160W as listed in Table 2 are underestimates of the flux caused by the presence of SN flux in the template images from Dickinson et al. The templates were obtained 177 days after the discovery epoch of 1997 December 23. To accurately correct the SN magnitudes in Table 2 for the oversubtraction, it is necessary to fit the light curve to determine this correction. This step is performed in $\S 3.2$.

\subsection{Host Spectroscopy}

On the nights of 2000 November 21, 26, and 27 UT, about $2 \mathrm{hr}$ of optical spectroscopy were obtained of the SN host using the echelle spectrograph and imager (ESI) on Keck II under poor conditions (seeing $\sim 1$ ".3 and an air mass of 1.7-2). During the nights of 2001 February 23 and $24 \mathrm{UT}, 3.5 \mathrm{hr}$ of optical spectroscopy were gathered using the low-resolution imaging spectrograph (LRIS; Oke et al. 1995 ) on Keck I in good conditions (seeing $\sim 0$ ".8 and an air mass of 1.4). An additional $2.5 \mathrm{hr}$ of optical spectroscopy were obtained with ESI on Keck II on the nights of 2001 February 26 and 27 UT. The total data set of optical spectroscopy consisted of $8 \mathrm{hr}$ with a wavelength coverage of 4000 to $10000 \AA$. A small amount of continuum flux was detected in the composite spectrum with evidence of some minor breaks in the galaxy SED, but there was no evidence of either a strong break in the galaxy SED or any emission lines.

Observations were made with the NIR spectrograph NIRSPEC (McLean et al. 1998) on Keck II on the nights of 2001 March 16 and 17 UT and on 2001 April 14 UT. On all dates, the 0 ".76 slit was oriented at a position angle (P.A.) of $184^{\circ} .6$ to include a galaxy $\sim 3^{\prime \prime}$ to the north.

In the March campaign, the slit was rotated to a P.A. such that light from both the host galaxy and the nearby bright galaxy fell onto the slit. The host galaxy was then positioned on the slit by first obtaining short-integration 


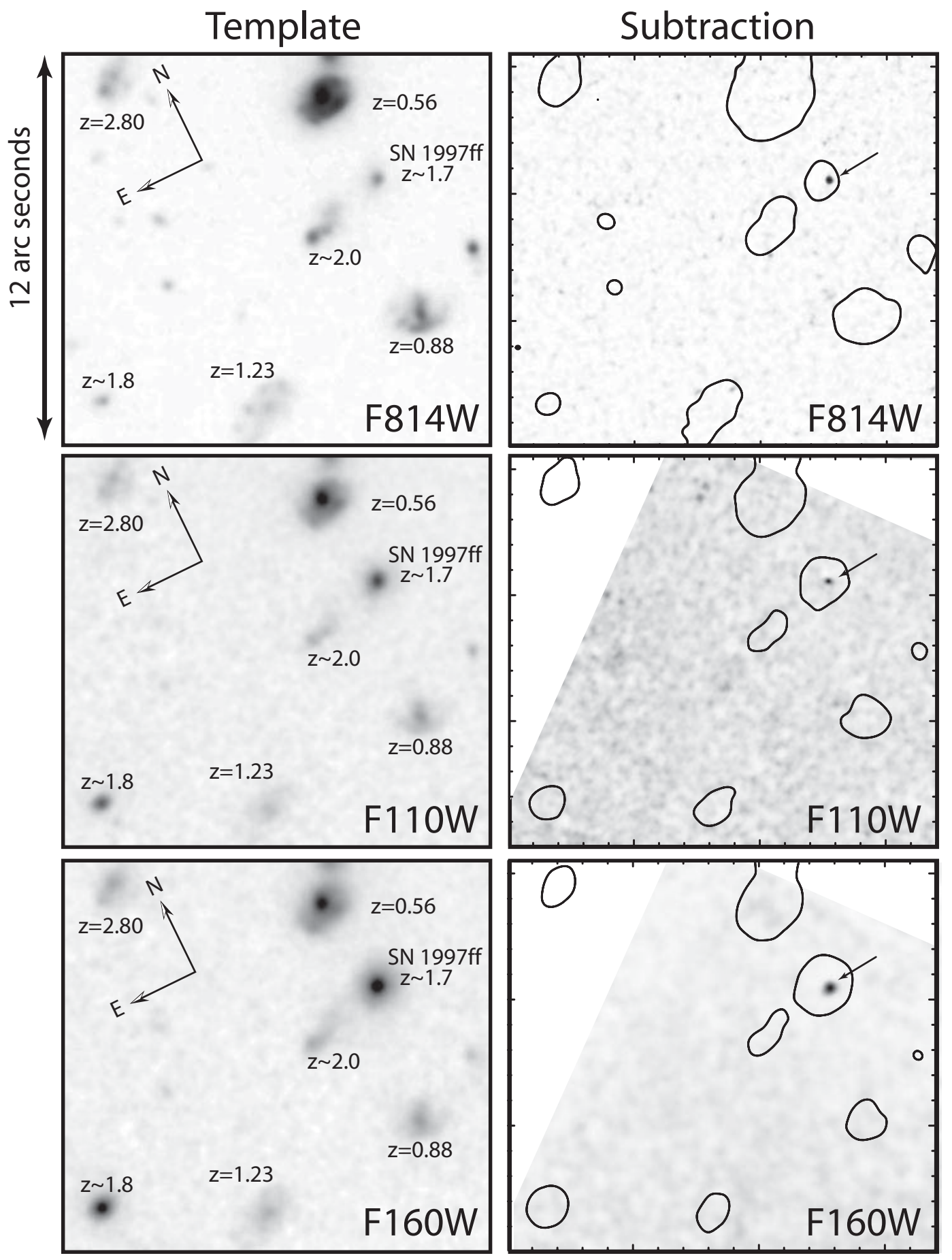

FIG. 3.- SN 1997ff in F814W $(I)$, F110W $(J)$, and F160W $(H)$. Images on the left show the region of the HDF-N near the SN host without the SN (template images). Images on the right show the difference in intensity between an SN image and the template image. Superimposed on this image are intensity contours. Spectroscopic redshifts (Cohen et al. 2000) are listed as exact while photometric redshifts (Budavári et al. 2000) are listed as approximate.

images of the field, then moving the telescope to position the nearby galaxy onto the slit. Spectroscopic observations were obtained by taking four $600 \mathrm{~s}$ integrations. After the first integration, the slit was moved $15^{\prime \prime}$ in the spatial direction on the array for the second integration, then moved back to the original position, where the process was repeated.

In the April campaign, the target was acquired by placing a nearby bright star onto the slit position desired and off- setting the telescope $\sim 85^{\prime \prime}$ to place the target at the same position on the slit. The conditions were excellent, with seeing estimated at 0".45 FWHM for the duration of the observation. Eight exposures of $900 \mathrm{~s}$ each were obtained; after each exposure, the offsets were reversed and the alignment of the star on the slit was checked using images obtained with the IR slit-viewing camera. Judging by these checks, the telescope offsetting and guiding was accurate to better than 1 pixel $(\sim 0$ ".14). For each exposure, the target 
TABLE 2

VeGa MagNitudes of SN 1997 $\mathrm{FF}^{\mathrm{a}}$

\begin{tabular}{|c|c|c|c|c|c|}
\hline Days since 1997 Dec 23 & $\begin{array}{c}\text { F814W } \\
\text { (mag) }\end{array}$ & $\begin{array}{c}\text { F110Wa } \\
\text { (mag) }\end{array}$ & $\begin{array}{c}\mathrm{F} 160 W^{\mathrm{a}} \\
\text { (mag) }\end{array}$ & $\begin{array}{c}K_{B}^{\mathrm{b}} \\
(\mathrm{mag})\end{array}$ & $\begin{array}{c}m_{\text {eff }}(B) \\
(\mathrm{mag})\end{array}$ \\
\hline-770 & $>27.3^{\mathrm{c}}$ & $\ldots$ & $\ldots$ & $\ldots$ & $\ldots$ \\
\hline-229. & $>26.0^{\mathrm{d}}$ & $\ldots$ & $\ldots$ & $\ldots$ & $\ldots$ \\
\hline $0.83 \ldots \ldots \ldots \ldots \ldots \ldots \ldots$ & $26.94(0.15)^{\mathrm{e}}$ & $\ldots$ & $\ldots$ & 0.96 & $25.98(0.15)$ \\
\hline $2.78 \ldots \ldots \ldots \ldots \ldots \ldots \ldots$ & $27.09(0.15)^{\mathrm{e}}$ & $\ldots$ & $\ldots$ & 1.01 & $26.08(0.15)$ \\
\hline $3.0 \ldots \ldots \ldots \ldots \ldots \ldots \ldots \ldots$ & $\ldots$ & $\ldots$ & $23.49(0.20)$ & -2.57 & $26.00(0.20)$ \\
\hline $3.74 \ldots \ldots \ldots \ldots \ldots \ldots \ldots$ & $26.92(0.13)^{\mathrm{e}}$ & $\ldots$ & $\ldots$ & 1.03 & $25.89(0.13)$ \\
\hline $10 . \ldots \ldots \ldots \ldots \ldots \ldots \ldots$ & $\ldots$ & $\ldots$ & $23.56(0.15)$ & -2.69 & $26.17(0.15)$ \\
\hline $14 . \ldots \ldots \ldots \ldots \ldots \ldots \ldots \ldots$ & $\ldots$ & $>23.3$ & $\ldots$ & -1.64 & $>24.86$ \\
\hline $28.5 \ldots \ldots \ldots \ldots \ldots \ldots \ldots$ & $\ldots$ & $\ldots$ & $23.84(0.20)$ & -3.14 & $26.83(0.20)$ \\
\hline $30.5 \ldots \ldots \ldots \ldots \ldots \ldots \ldots$ & $\ldots$ & $\ldots$ & $24.26(0.25)$ & -3.20 & $27.30(0.25)$ \\
\hline $32.0 \ldots \ldots \ldots \ldots \ldots \ldots \ldots$ & $\ldots$ & $25.67(0.30)$ & $\ldots$ & -1.65 & $27.16(0.30)$ \\
\hline $32.5 \ldots \ldots \ldots \ldots \ldots \ldots \ldots$ & $\ldots$ & $\ldots$ & $24.27(0.20)$ & -3.26 & $27.36(0.20)$ \\
\hline $34.5 \ldots \ldots \ldots \ldots \ldots \ldots \ldots$ & $\ldots$ & $25.67(0.20)$ & $\ldots$ & -1.66 & $27.14(0.20)$ \\
\hline $37.0 \ldots \ldots \ldots \ldots \ldots \ldots \ldots$ & $\ldots$ & $25.92(0.20)$ & $\ldots$ & -1.66 & $27.38(0.20)$ \\
\hline
\end{tabular}

${ }^{a}$ Underestimates caused by flux in template observed at +177 days.

${ }^{\mathrm{b}} K$-corrections to the $B$ band for best fit: $z=1.7$, SN discovered one week past maximum brightness.

c Estimated from initial HDF-N.

d Estimated from HST archive images (GO 7588).

e From GNP99.

was placed on a different position along the $42^{\prime \prime}$ slit to reduce detector systematics and to allow for more accurate sky subtraction.

All the data were reduced following a procedure very similar to that described in Pettini et al. (2001). The data from 2001 March and 2001 April were reduced independently and then combined with appropriate weighting into the final co-added two-dimensional spectrum. The onedimensional spectrum was extracted using an aperture of $1 " 2$.

The feature which may be very tentatively identified as the [O II] $3727 \AA$ Aine at $z=1.755$ falls in a relatively rare region that is unaffected by bright $\mathrm{OH}$ lines in the night sky, although there is a strong sky emission feature at $1.029 \mu \mathrm{m}$, i.e., just to the red of the putative $[\mathrm{O}$ II] feature. The excess emission is present in individual subsets of the data and becomes more prominent when the 40 minutes of integration time from 2001 March are combined with the 120 minutes from 2001 April. The line is well resolved at a spectral resolution of $\sim 7.5 \AA$, consistent with expectations for the $[\mathrm{O}$ II] doublet, which has a rest-frame separation of $\sim 2$ $\AA$. (Single emission lines in the spectrum of the nearby $z=0.556$ galaxy, and most but not all sky-subtraction residuals, are significantly narrower.) This feature is noteworthy and may function as a useful hypothesis to test with future observations, but at this time its validity is highly uncertain.

\section{ANALYSIS}

\subsection{The Redshift of the Supernova and Its Host}

As discussed in $\S 2$, fitting the $U_{300} B_{450} V_{606} I_{814} J_{110}$ $H_{160}$ space-based photometry and the $J_{125} H_{165} K_{s}$ ground-based photometry of 4-403.0 to galaxy SEDs yields a photometric redshift of $z=1.55$ to 1.70 with variations depending on whether the fitted model is based on template galaxy SEDs (Coleman, Wu, \& Weedman 1980, hereafter CWW) or galaxy eigenspectra (Budavári et al. 2000). These fits can be seen in Figure 4. The well-constrained fits between model and data indicate a far greater degree of precision in the redshift $(1 \sigma \approx 0.02)$ than is empirically found by comparing photometric and spectroscopic redshifts. We therefore consider the empirical dispersion from Budavári et al. (2000) as the measure of the individual uncertainty.

Using a set of orthogonal eigenspectra derived from the CWW galaxy template SEDs yields the lowest redshift, $z=1.55 \pm 0.15$, and empirically the least precise and robust (Budavári et al. 2000). Indeed, significant outliers occasionally result from the application of this method. A more robust and precise redshift estimate comes from the fitting of improved eigenspectra. These are derived from the CWW SED eigenspectra, which are first "repaired" (see Budavári et al. 2000) as required to improve the agreement between the photometric and spectroscopic redshifts. Mild repairing yields $z=1.65 \pm 0.13$ (model KL2) and further repairing (model KL5) provides $z=1.70 \pm 0.10$ (Budavári et al. 2000). The biggest advantage of first repairing the eigenspectra is the suppression of outliers, yielding a more robust estimate. In addition, the relatively simple SEDs of early-type galaxies such as 4-403 generally provide more robust photometric redshifts (Budavári et al. 2000). For 4-403.0, we will adopt $z=1.65 \pm 0.15$ as a measurement that is representative of the photometric redshift.

An additional and independent pathway to determine the redshift is from the $\mathrm{SN}$ colors. For the following analysis, we will provisionally adopt the classification from GNP99 of SN $1997 \mathrm{ff}$ as a Type Ia supernova based on its red, elliptical host galaxy. However, in $\S 4.1$ we will analyze the degree to which this classification is merited.

As can be seen in Table 2, coincident or near-coincident measurements of SN $1997 \mathrm{ff}$ in different bands provide an observed $I-H$ color of $3.5 \pm 0.2 \mathrm{mag}$ and a $J-H$ color of $1.6 \pm 0.2 \mathrm{mag}, 30 /(1+z)$ days later in the rest frame. In Figure 5, we plot these measurements as a function of expected colors of SNe Ia over their temporal evolution at different redshifts (note that the size of the point scales with the temporal proximity to $B$ maximum). SNe Ia are bluest shortly after explosion and become redder with age. They 


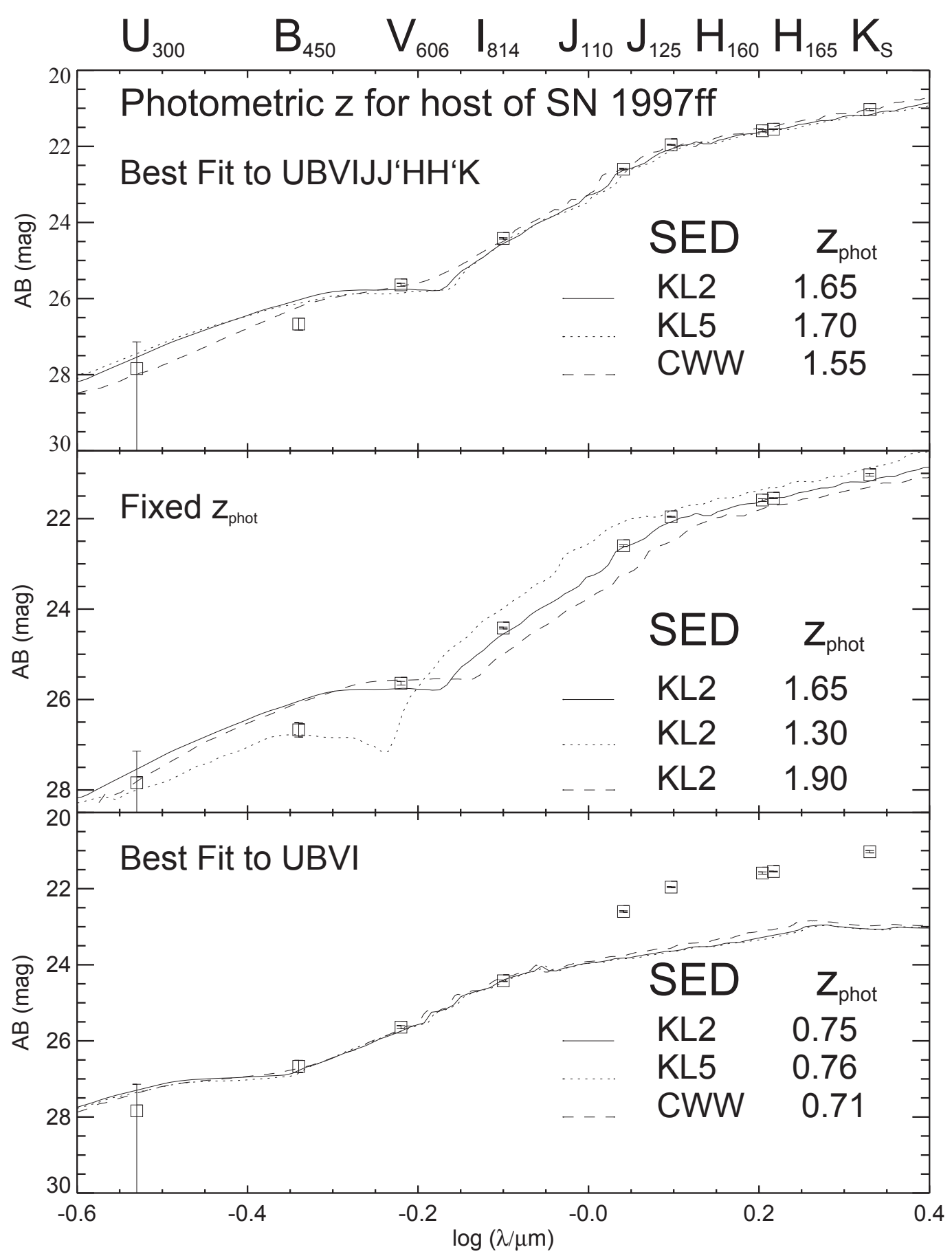

FIG. 4.-Photometric redshift estimate for the host of SN 1997ff. This estimate employs six photometric magnitudes from $H S T$ observations and three from ground-based observations (Budavári et al. 2000). Galaxy magnitudes are given in Table 1. The top panel shows the best fits for the CWW (galaxy SEDs), KL2 (eigenspectra), and KL5 (eigenspectra) models. The middle plot shows the sensitivity of the fit to the redshift. The bottom plot shows the biases that result from using only the WFPC2 data.

reach their reddest color $\sim 25$ days after maximum in these bandpasses and return to a modestly bluer color during the subsequent nebular phase. As seen in Figure 5, either of the observed colors of SN 1997ff is redder than an SN Ia at any phase for $z<1$. The $I-H$ color sets a limit of $z>1.2$ while the $J-H$ color is more stringent with $z>1.4$, both at the greater than $95 \%$ confidence level.

However, the constraint obtained from each observed color treated independently is less restrictive than if we con- sider their separation in time. In this case, our lower limit on the redshift comes from assuming that the later color measurement occurs at the reddest phase of the SN and from requiring the earlier color measurement to be consistent with an earlier, bluer phase of the SN. In this way we find $z>1.45$ at the $95 \%$ confidence level. An upper limit on the redshift comes from the colors and the observation that the $\mathrm{SN}$ is declining during the five F160W measurements by $\sim 1$ mag in the $\sim 30$ observed-frame days. As seen in Figure 


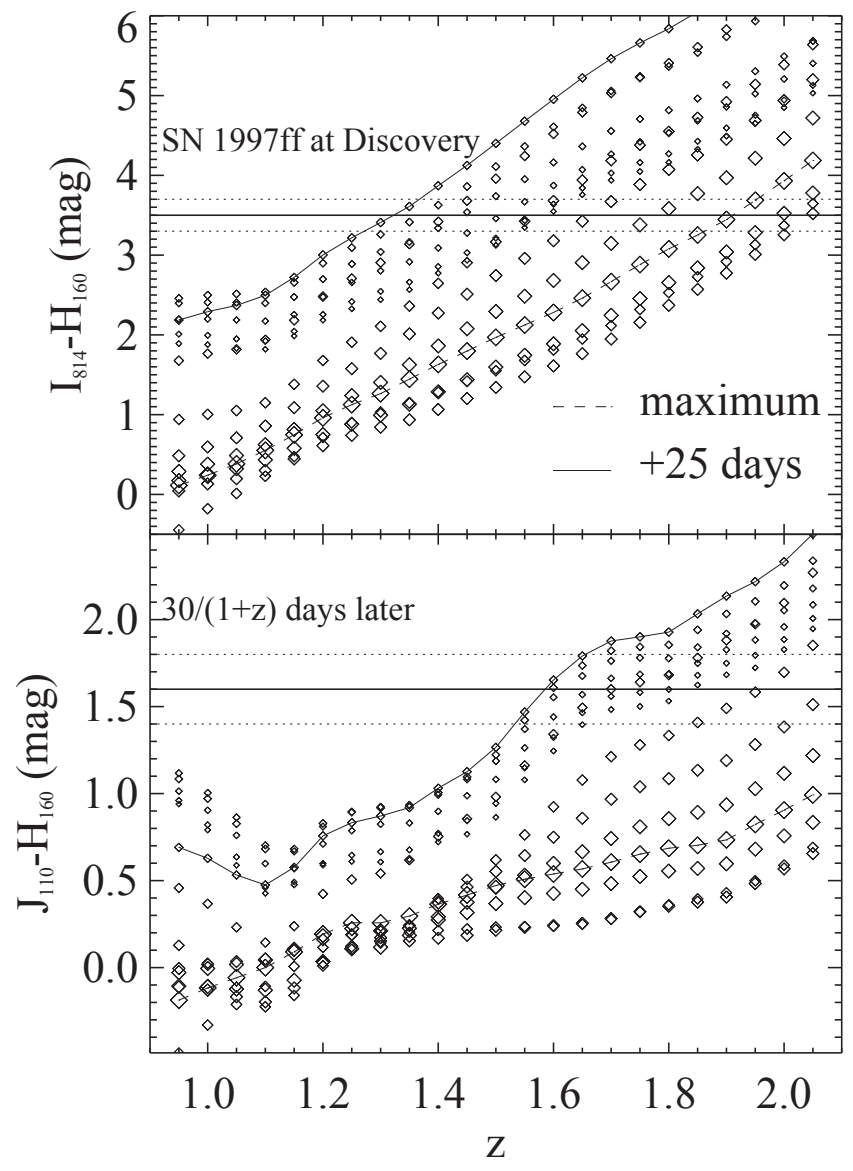

FIG. 5.-Comparison between the observed and expected NIR colors of an SN Ia as a function of assumed redshift. For a given redshift, the color evolution is plotted beginning 15 days before maximum (bluest point) and again in 5 day intervals. SNe Ia redden with time, reach their reddest color at 25-30 days after maximum, and then become bluer by a few tenths of a magnitude. The relative size of the point scales with temporal proximity to $B$ maximum. The observed colors of SN $1997 \mathrm{ff}$ at discovery and 30/(1 $+z)$ days later puts strong constraints on the redshift and age of the SN.

5 , the redshift of the SN must be less than 2.0 to both match the $I-H$ color and be discovered on the decline. A redshift in the range of $1.5<z<1.8$ is coarsely consistent with the observed colors and temporal behavior.

This simple analysis assumes negligible reddening from the host. While this assumption is appropriate for most elliptical hosts, we will consider the effect of reddening explicitly in $\S 4.2$. Note that Galactic reddening is very low toward the HDF-N.

The above exercise cannot be performed readily if we assume the SED of a common SN II instead of an SN Ia. Not surprisingly, a comparison of the observed colors of SN $1997 \mathrm{ff}$ to those expected for a blue SN II (similar to the well-observed SN 1979C; e.g., Schmidt et al. 1994) yields poor fits to both color measurements and their time separation. For any value of the redshift, the observed $J-H$ color is far bluer than the color of a common SN II-P or SN II-L (for definitions, see Barbon, Ciatti, \& Rosino 1979) at a phase dictated by the requirement of matching the earlier $I-H$ color. A common SN II would not match both color measurements of SN $1997 \mathrm{ff}$ unless it were observed shortly after explosion and at $z \approx 2$. However, the observed decline of SN 1997ff appears inconsistent with the expected rising luminosity or plateau phase of an SN II shortly after explosion. Reddening of the $\mathrm{SN}$ would result in an even greater difference between the data and a common SN II. Based on the observed colors and declining luminosity alone, the identification of SN $1997 \mathrm{ff}$ as a normal SN II is strongly disfavored, a conclusion which is discussed further in $\S 4.2$.

The blue color, bright magnitude, and observed decline of SN 1997ff might be consistent with some SNe IIn (Filippenko 1997), which show considerable heterogeneity in their light curves (Schlegel 1990; A. V. Filippenko 2001, private communication). However, these objects are rare, and they are not found in old stellar populations $(\S 4.2)$. Similarly, SN $1997 f f$ was unlikely to be an SN Ib or SN Ic, which occur in very young stellar populations, are generally redder than SN 1997ff, and are rare (see $\S 4.2$ ).

The spectroscopy of the host presented in $\S 2.4$ provides some evidence that is consistent with the preceding redshift determinations and potentially more precise but currently unreliable. As can be seen in Figure 6 (top panel), the optical spectrum of the host suggests two minor breaks that could be identified with the rest-frame breaks at 2640 and $2900 \AA$ because of blends of metals (Spinrad et al. 1997). A simple $\chi^{2}$

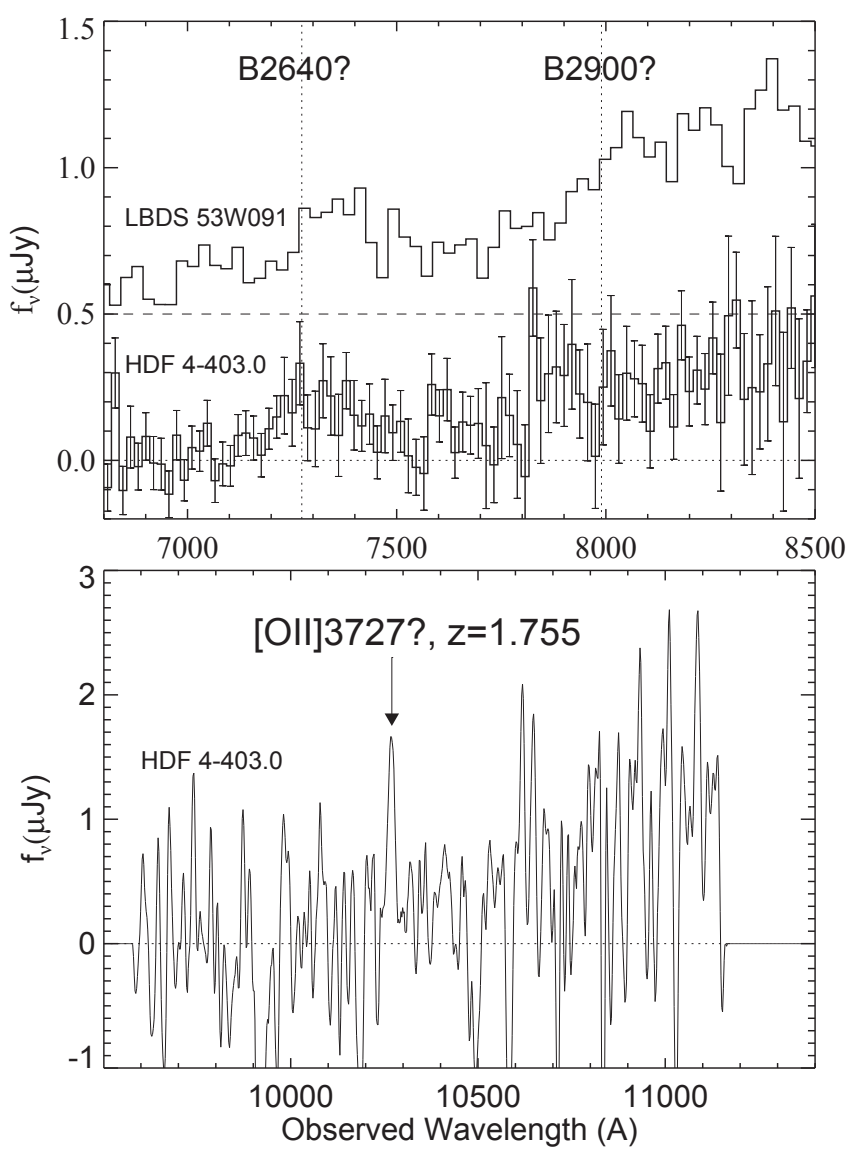

FIG. 6.-Optical and NIR spectroscopy of the host, HDF 4-403.0, from the Keck telescope. The top panel shows the optical spectra of the SN host compared to the spectrum of an old, red elliptical galaxy, LBDS 53w091 $\left(z=1.55\right.$; Spinrad et al. 1997), transformed to $z=1.755$. A simple $\chi^{2}$ minimization provides a possible match at a redshift for the SN host of $z=1.67$ to 1.79 , but this match is not robust. The bottom panel shows the NIR spectroscopy of the host. An apparent weak emission line, if identified as [O $\mathrm{II}] \lambda 3727$, would yield $z=1.755$, but this redshift determination is tentative. A gradient in the detected continuum is apparent, with an increase to the red. 
minimization between the optical spectrum of the host and the same region of the SED of the $z=1.55$ elliptical LBDS 53w091 (Spinrad et al. 1997) yields a significant minimum bounded by $z=1.67$ and 1.79 ( $3 \sigma$ confidence level). However, this minimum does not appear robust and we cannot rule out the possibility that other redshift matches are possible given other models for the host SED.

The shape of the extracted continuum (Fig. 6b) is consistent with the broadband photometry from HST WFPC-2 and NICMOS observations and is not inconsistent with the presence of a break at $4000 \AA$ in the galaxy rest frame (for $z \approx 1.7$ ), although for a possible redshift of $z \approx 1.8$, the region longward of the $4000 \AA$ break is beyond the wavelength range covered by the NIRSPEC spectrum.

Although the spectroscopic redshift indicators are suggestive of a match with the photometric indicators, the quality of the spectra is too low and the identification of spectral features too uncertain to reach a robust determination of the redshift from the spectroscopy alone. Therefore in the following section we derive constraints from the $\mathrm{SN}$ without employing the spectroscopic redshift indications.

\subsection{Probability Density Functions for SN $1997 f f$}

The simple method for constraining the redshift described in the previous section can be refined to make use of all of the SN photometric data simultaneously. By varying the parameters needed to empirically fit an SN Ia, such as the light-curve shape, distance, redshift, and age, we can use the quality of the fit to determine the probability density function (PDF) of these parameters. An additional component of this fitting process is to include the known correlation between SN Ia light-curve shapes and their peak luminosities (Phillips 1993; Phillips et al. 1999; Riess, Press, \& Kirshner 1996; Perlmutter et al. 1997). Examples of this fitting process can be seen in Figure 7 as applied to SN $1997 \mathrm{ff}$.

In Appendix A, we develop a simple formalism for using the observations of SN 1997ff and any prior information that is appropriate to determine the PDF of the parameters of luminosity, distance, redshift, and age commonly used to empirically model SNe Ia. This method is quite general and its application to $\mathrm{SN}$ Ia photometry is equivalent to the use of a common light-curve fitting method, such as $\Delta m_{15}(B)$

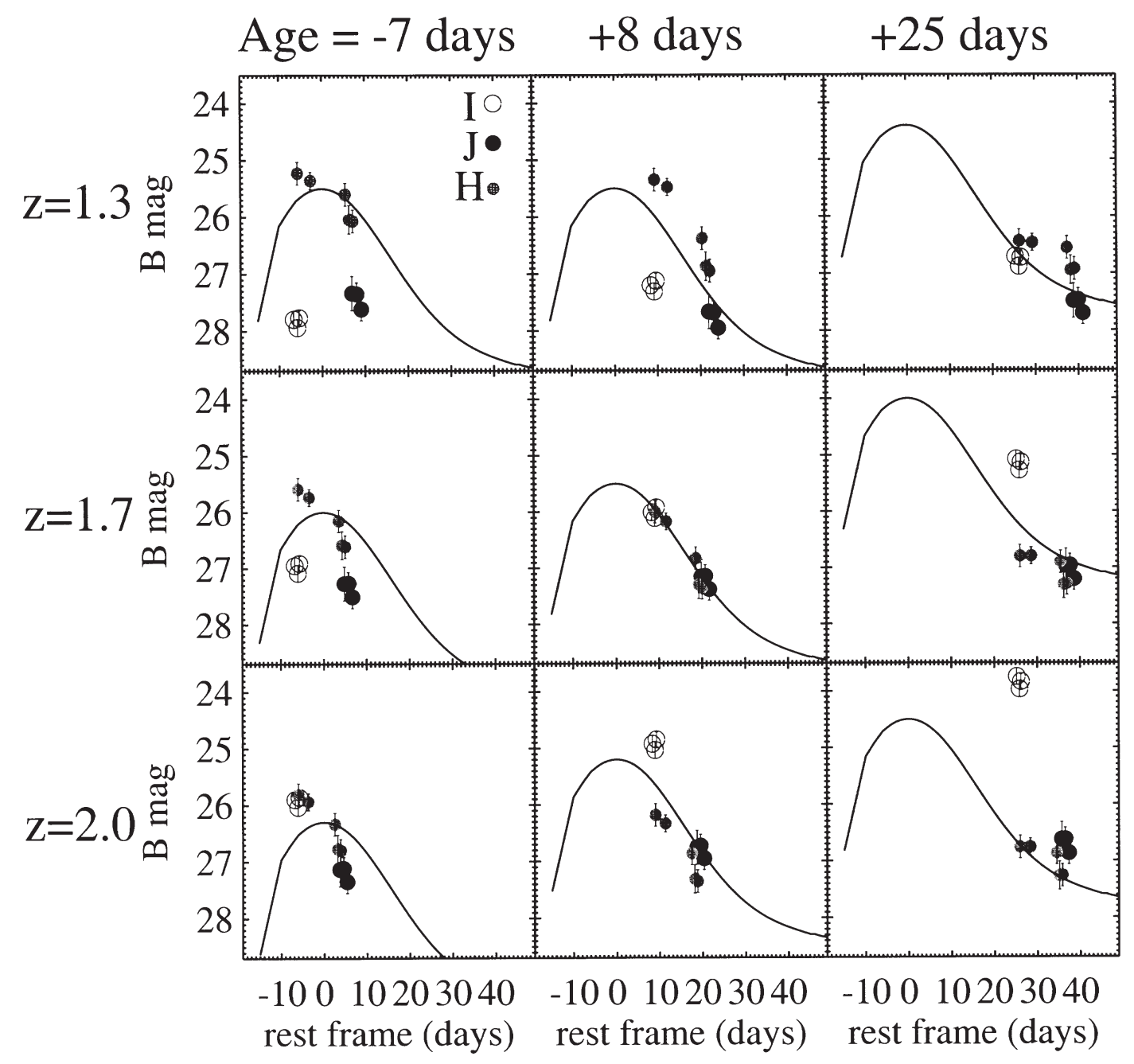

Fig. 7.-Comparison between the $B$-band light curve of a normal SN Ia and the observed data transformed to rest-frame $B$ for different assumed redshifts and discovery ages. The observed SN colors, or, for the transformation to a fixed bandpass shown here, the $K$-corrections are a strong function of redshift and SN age. The distance modulus may be constrained by offsetting the model light curve in magnitudes. A good fit between model and data occurs only in a narrow range of redshifts and ages as shown in the middle panel. 
(Phillips et al. 1999; Hamuy et al. 1996), the Multicolor Lightcurve Shape method (MLCS; Riess et al. 1996, 1998), or the "stretch method" (Perlmutter et al. 1997), in cases for which the light-curve information is more constraining than prior information. The advantage of this method is its ability to incorporate prior information (e.g., a photometric redshift) in a statistically sound way that properly assigns weights to the relative constraints provided by data and priors.

Given the presence of SN light in the M. Dickinson et al. (2001, in preparation) template images taken 177 days after the SN discovery, it is necessary to restore the flux that is necessarily oversubtracted from the F110W and F160W magnitudes listed in Table 2. The size of the correction depends on the redshift, age of discovery, and shape of the SN light curve. Therefore, we implemented this correction during the process of fitting the data to parameterized models. In the case of the best-fitting light curve, the underestimate of the SN magnitudes from the GTO campaign is 0.1 to 0.2 mag (depending on the phase), and for the guide star test exposures (taken when the $\mathrm{SN}$ is $\sim 1$ mag brighter) the correction is $0.05 \mathrm{mag}$.

We determined the PDF for SN $1997 \mathrm{ff}$ using the methods outlined in Appendices A and B, the previously described data, and specific priors we discuss here.

Riess et al. (1998) found that the observed peak $B$-band luminosities of SNe Ia at low redshift $(0.01<z<0.1)$ and high redshift $(0.3<z<1.0)$ are characterized by distribution functions with $\sigma_{M} \leq 0.25 \mathrm{mag}$. An even narrower luminosity function of $\sigma_{M}=0.17 \mathrm{mag}$ for $\mathrm{SNe}$ Ia was found by Perlmutter et al. (1999) for a similar set of low-redshift SNe Ia and an independent set of high-redshift SNe Ia. Although the peak luminosity function of very nearby $\mathrm{SNe}$ Ia $(z<0.01)$ includes a low-luminosity "tail" populated by so-called SN 1991bg-like SNe Ia (Filippenko et al. 1992b; Leibundgut et al. 1993; Modjaz et al. 2001), such SNe which are dimmer at peak by $\sim 2$ mag are undetected in highredshift, magnitude-limited surveys (Li, Filippenko, \& Riess 2001). We defined a normal function prior for the observed peak luminosity of SNe Ia with a standard deviation of 0.25 mag. Assuming that SN 1997ff was drawn from the same population of SNe Ia that lower redshift $\mathrm{SNe}$ Ia have sampled, we expect this prior to be valid. If, however, the luminosities of SNe Ia have significantly evolved by $z \approx 1.7$, then this should be apparent in the divergence of the redshift-magnitude relation of $\mathrm{SNe}$ Ia from cosmological models. We also considered a much less constraining prior of $\sigma_{M}=0.50 \mathrm{mag}$. Though this prior underutilizes our empirical knowledge of SN Ia luminosities, it does provide a wider latitude to allow the photometry of SN $1997 \mathrm{ff}$ to constrain the fit to its model light-curve shape. (Quantitatively, this prior yields similar results as a perfectly flat luminosity prior.) It also provides for a possibly larger dispersion in peak luminosities of $\mathrm{SNe}$ Ia at higher redshifts.

As provided in $\S 3.1$, the photometric redshift of the host galaxy from space-based $U_{300} B_{450} V_{606} I_{814} J_{110} H_{160}$ photometry and the ground-based $J_{125} H_{165} K_{s}$ photometry results in the prior constraint $z=1.65 \pm 0.15$ (Budavári et al. 2000). (This constraint is also consistent with the host spectroscopy as presented in $\S 3.1$.) We determined the PDF of SN 1997ff both with and without this prior photometric constraint. The latter approach, while not optimal for determining the best constraints, does allow us to determine the $\mathrm{SN}$ redshift solely from the $\mathrm{SN}$ data and test its compatibility with the photometric and spectroscopic redshift determinations.

Finally, because no additional information is available to constrain the remaining two SN Ia parameters, distance and age at discovery (see Appendix A), no further knowledge of these variables was included in the priors.

By marginalizing the four-dimensional PDF for SN $1997 \mathrm{ff}$ over any three parameters, we determined the PDF for the fourth parameter of interest. The marginal probability for the redshift, age at discovery, and luminosity are shown in Figure 8. The marginalized PDF of the redshift is not a simple function, though it is strongly peaked near $z \approx 1.7$ and is insignificant outside the range $1.4<z<1.95$. A much lower local maximum is seen at $z=1.55$. The redshift measurement of SN $1997 \mathrm{ff}$ can be crudely approximated by $z=1.7_{-0.15}^{+0.10}$.

The consistency of the three redshift indicators, determined independently from the galaxy colors, the supernova colors, and the host spectroscopy, provides a powerful and successful cross-check of our redshift determination. Excluding any galaxy redshift information has little impact on the marginalized redshift PDF of the SN because the SN data are significantly more constraining for the redshift determination. The cause of the difference in measured photometric redshift precision lies in the difference in the relative homogeneity of galaxy and SN Ia colors. For the galaxy photometric determination, the precision of this method is limited by the variations of galaxy SEDs beyond those which can even be accounted for from the superposition of eigenspectra. In contrast, $\mathrm{SNe}$ Ia colors are far more homogeneous and their mild inhomogeneities are well characterized, leading to more precise constraints on the photometric redshift.

From the redshift determination, we conclude that SN $1997 \mathrm{ff}$ is the highest redshift SN observed to date (as suspected by GNP99), easily surpassing the two $\mathrm{SNe}$ Ia at $z=1.2$ from the HZT (Tonry et al. 1999; Coil et al. 2000) and the SCP (Aldering et al. 1998). The statistical confidence in this statement is very high.

Marginalizing the PDF for SN 1997ff over the age-ofdiscovery parameter yields the function shown in Figure 8. We conclude that the SN was discovered by Gilliland \& Phillips (1998) at an age of a week past $B$-band maximum with an uncertainty of $\sim 5$ days; however, this estimate is highly non-Gaussian, as can be seen in Figure 8. An additional, local maximum in the marginalized probability is evident at an age of $\sim 15$ days after maximum. The possibility that the $\mathrm{SN}$ was discovered at this later age corresponds to the same model for which the weaker maximum in the redshift PDF indicated that $z=1.55$. This correlation between the redshift and age parameters is a natural consequence of the reddening of an SN Ia as it ages and is shown in Figure 9.

Little additional constraint on the peak-luminosity/lightcurve-shape parameter is gained from the fit, beyond what is provided by the luminosity function prior as seen in Figure 8. The $B$-band light curve of a typical SN Ia (e.g., Leibundgut 1988; $\Delta m_{15}(B)=1.1 \mathrm{mag}$ from Hamuy et al. 1996; $\Delta=0$ mag from Riess et al. 1998) provides an excellent fit to the SN 1997ff data when the other three parameters are set to their most likely values, as in the middle panel of Figure 7. By relaxing the prior to $\sigma_{M}=0.50 \mathrm{mag}$, we can better determine the degree to which the $\mathrm{SN}$ fit constrains 

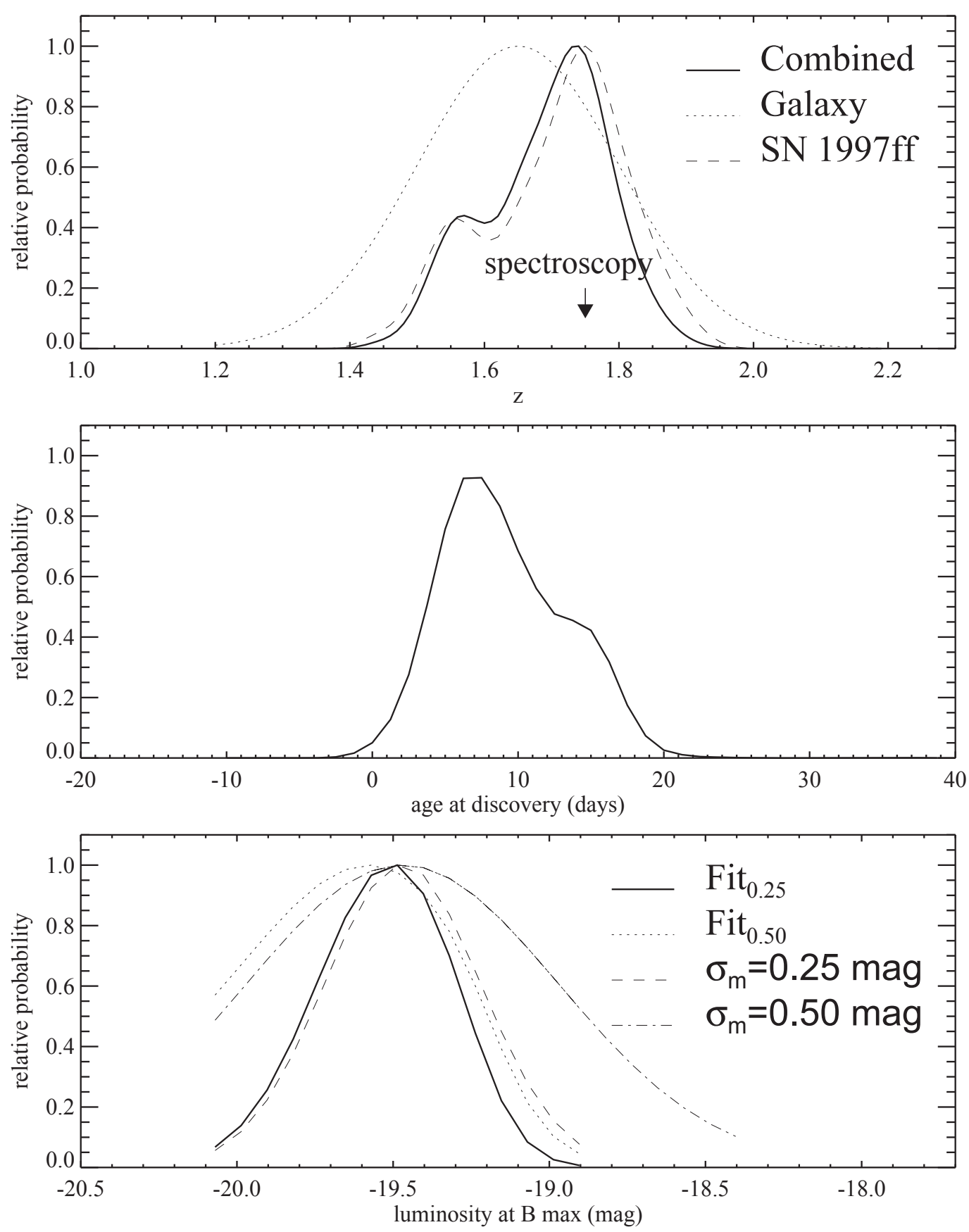

FIG. 8.-Marginalized probability density functions for SN Ia model parameters used to fit SN 1997ff. The top panel shows the PDF for the redshift constraints from the galaxy photometry, from the $\mathrm{SN}$, and from both sources. The middle panel shows the constraint on the age of the discovery relative to $B$ maximum. The bottom panel shows two forms of the observed luminosity function for SNe Ia and the constraints on the luminosity of SN $1997 \mathrm{ff}$ using the priors and the light-curve fits.

its possible light-curve shape and, hence, its correlated peak luminosity. As shown in Figure 8, the fit to the SN 1997ff data disfavors a fast declining, subluminous SN Ia lightcurve shape. Quantitatively, this result excludes the hypothesis that SN $1997 \mathrm{ff}$ is as much as $0.5 \mathrm{mag}$ subluminous at peak.

On the bright limb of the peak-luminosity/light-curveshape relationship, the constraints are nearly parallel to the luminosity function prior. The bright limb is defined by spectroscopically peculiar SNe Ia known as SN 1991T-like events (Filippenko et al. 1992a; Phillips et al. 1992; Li et al.
1999), which may be overluminous by $0.3-0.6$ mag. It is important to note that SNe Ia are neither observed nor expected to reach peak magnitudes of more than $\sim 0.6 \mathrm{mag}$ brighter than the typical (Hamuy et al. 1996; Höflich \& Khokhlov 1996). Therefore, we do not consider light-curve shape models that are extrapolated beyond the most luminous SNe Ia observed locally.

Of critical importance to cosmological hypothesis testing is the determination of the luminosity distance to such highredshift SNe Ia. Because of the significant correlation between the distance and photometric redshift parameters 


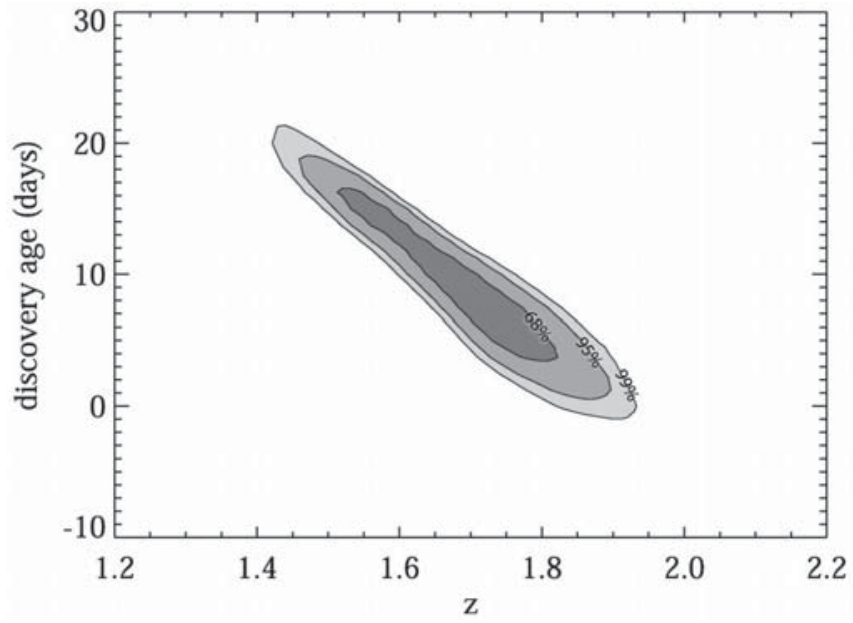

FIG. 9.- Confidence intervals for the discovery age (days relative to $B$-band maximum) and redshift of SN 1997ff. Because the observed SN colors are a strong function of both of these parameters, a high degree of correlation exists in their simultaneous determination.

and the need to use both of these parameters for cosmological applications, we determined the two-dimensional PDF for distance and redshift simultaneously. This function is shown in Figure 10 for the different priors described above. Although it is preferable to include prior information from the galaxy photometric redshift estimate and the observed luminosity function of $\mathrm{SNe}$ Ia, the constraints shown in the distance-redshift plane are only minimally improved with this information, and our subsequent conclusions are insensitive to these priors.

We also determined the likelihood function for the distance assuming the tentative spectroscopic redshift. We find $m-M=45.15 \pm 0.34$ mag. This likelihood function is quite Gaussian within the $2 \sigma$ boundaries but flattens beyond for shorter distances (corresponding to an older discovery age) and steepens beyond for longer distances

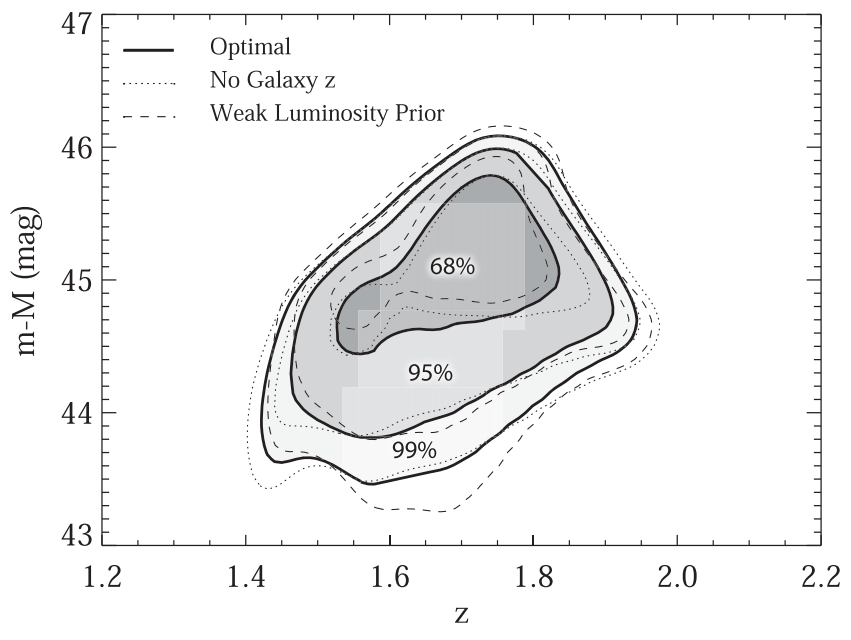

FIG. 10.-Confidence intervals for the distance modulus $(m-M)$ and redshift of SN 1997ff. The intervals were calculated using the galaxy photometric redshift and the observed luminosity function of SNe Ia (optimal), neglecting the galaxy photometric redshift and using a weak prior on SN Ia luminosities described in the text.

(corresponding to a discovery near maximum). If we assume the tentative spectroscopic redshift, we find the age of discovery to be $6 \pm 2$ days after $B$-band maximum and tighter constraints on the peak-luminosity/light-curve-shape parameter. For the latter, we find the SN to be $0.05 \pm 0.20$ mag fainter at peak than average, which makes it a highly typical supernova.

\subsection{Cosmological Constraints}

In Figure 11 we show the redshift and distance data (i.e., the Hubble diagram) for SNe Ia as presented by the Supernova Cosmology Project (Perlmutter et al. 1999) and the High-z Supernova Search Team (Riess et al. 1998). These data have been binned in redshift to depict the statistical leverage of the SN Ia sample. Overplotted are the cosmo-

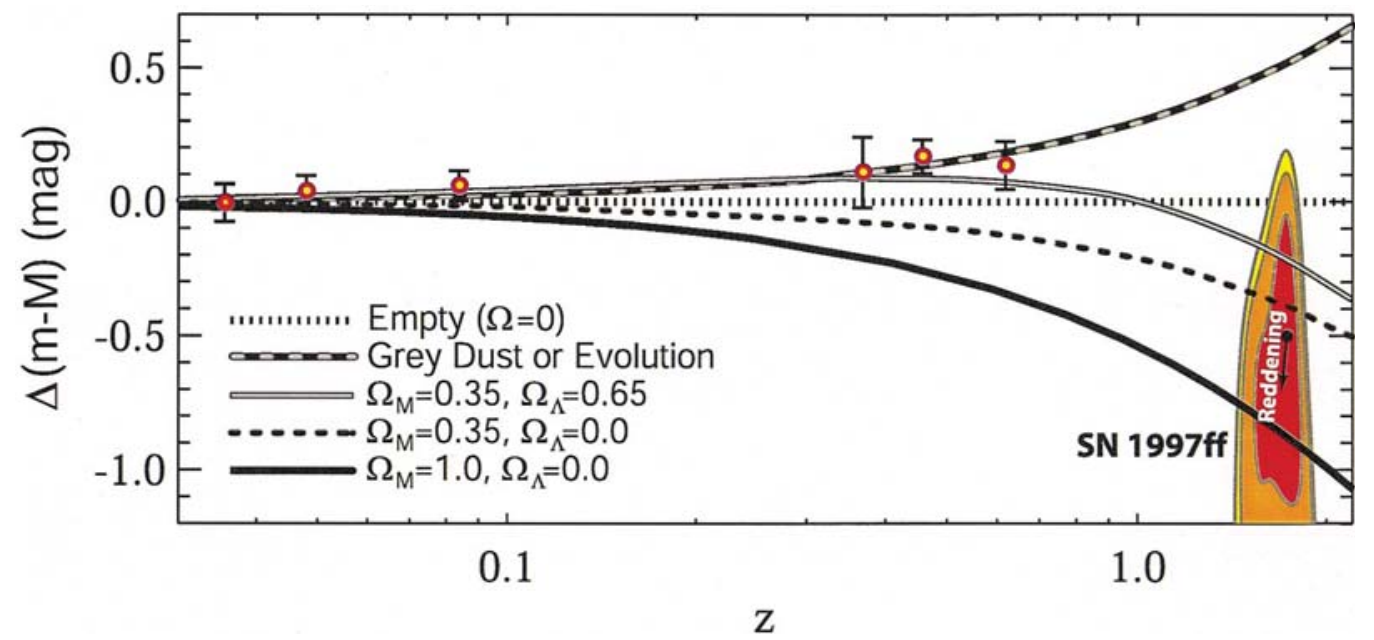

FIG. 11.-Hubble diagram of SNe Ia minus an empty (i.e., "empty" $\Omega=0$ ) universe compared to cosmological and astrophysical models. The points are the redshift-binned data from the HZT (Riess et al. 1998) and the SCP (Perlmutter et al. 1999). Confidence intervals (68\%, 95\%, and 99\%) for SN 1997ff are indicated. The modeling of the astrophysical contaminants to cosmological inference, intergalactic gray dust or simple evolution, is discussed in $\$ 4.2$. The measurements of SN $1997 \mathrm{ff}$ are inconsistent with astrophysical effects that could mimic previous evidence for an accelerating universe from SNe Ia at $z \approx 0.5$. 
logical models $\Omega_{M}=0.35, \Omega_{\Lambda}=0.65$ (favored), $\Omega_{M}=$ $0.35, \Omega_{\Lambda}=0.0$ (open), $\Omega_{M}=1.00, \Omega_{\Lambda}=0.0$ (Einstein-de Sitter), and an astrophysical model representing a progressive dimming in proportion to redshift caused by gray dust or simple evolution within an open cosmology. This model is further described in $\S 4.2$. All data and models are plotted as their difference from an empty universe $\left(\Omega_{M}=0.0, \Omega_{\Lambda}=\right.$ 0.0 ).

All models are equivalent in the limit of $z=0$. Differences in the models are considerable and detectable at $z>0.1$. Evidence for a significant dark-energy density and current acceleration is provided by the excessive faintness of the binned data, with $0.3<z<0.8$ compared with that of the open model, yielding a net difference of $\sim 0.25 \mathrm{mag}$.

The lack of SNe Ia at an independent redshift interval, beyond $z=1$, provides only the slimmest of margins for inferring the need for dark energy. An alternative explanation for the faintness of SNe Ia at $z \approx 0.5$ is a contaminating astrophysical effect. Two often-cited candidates for these effects are $\mathrm{SN}$ evolution and gray intergalactic dust. Although direct tests for these effects have thus far yielded little evidence to support either (Riess 2000; Riess et al. 2000), the standard of proof for accepting vacuum energy (or quintessence) is high. A more powerful test for any astrophysical effect that continues to $\operatorname{dim} \mathrm{SNe}$ at ever greater redshifts is to observe SNe Ia at $z>1$ (Filippenko \& Riess 2000). At these redshifts, the universe was more compact and familiar gravity would have dominated cosmological repulsion. The resulting deceleration at these redshifts would be apparent as a brightening of $\mathrm{SNe}$ Ia relative to a coasting cosmology or to the aforementioned astrophysical effects. The redshift of SN $1997 \mathrm{ff}$ is high enough to probe this earlier epoch and, together with the distance measurement, provides the means to discriminate between these hypotheses.

In Figure 11 we show the constraints derived from SN $1997 \mathrm{ff}$. In the redshift-distance plane, the principal axes of the error matrix from the photometric analysis are not quite perpendicular and the confidence contours are complex. Because there is only one object available in this highest redshift interval, we prefer to interpret Figure 11 with broad brush strokes.

SN 1997ff is brighter by $\sim 1.1 \mathrm{mag}$ (and therefore closer) than expected for the persistence of a purported source of astrophysical dimming at $z \approx 0.5$ and beyond. The statistical confidence of this statement is high $(>99.99 \%)$. This conclusion supports the reality of the measured acceleration of the universe from $\mathrm{SNe}$ Ia at $z \approx 0.5$ by excluding the most likely, simple alternatives. To avoid this conclusion requires the addition of an added layer of astrophysical complexity (e.g., intergalactic dust that dissipates in the interval $0.5<z<1.7$ or luminosity evolution that is suppressed or changes sign in this redshift interval). Other astrophysical effects, such as a change in the SN Ia luminosity distance caused by a change in metallicity with redshift, are also disfavored (Shanks et al. 2001). Systematic challenges to these conclusions are addressed in $\S 4$.

Other cosmological models that predict a relative dimming of SNe Ia at $z>1$, such as the "quasi-steady state hypothesis," appear to be in disagreement with this observation (Banerjee et al. 2000; Behnke et al. 2001). Similarly, models with relatively high vacuum energy and relatively low mass density are excluded (e.g., $\Omega_{\Lambda} \approx 1, \Omega_{M} \approx 0$ ). If we assume an approximately flat cosmology, as required by observations of the CMB, and a cosmological constant-like nature for dark energy, the observations of SN 1997ff disfavor $\Omega_{\Lambda}>0.85$ or, alternately, $\Omega_{M}<0.15$.

SN 1997ff also provides an indication that the universe was decelerating at the time of the supernova's explosion. To better understand this likelihood, in Figure 12 we show the redshift-distance relation of SNe Ia compared to that of a family of flat, $\Omega_{\Lambda}$ cosmologies. For such cosmologies, the transition redshift between the accelerating and decelerating epochs occurs at a redshift of $\left[2 \Omega_{\Lambda} / \Omega_{M}\right]^{1 / 3}-1$ (M. Turner, 2001, private communication). For increasing values of $\Omega_{\Lambda}$, the transition point (i.e., the coasting point) occurs at increasing redshifts. The highest value of $\Omega_{\Lambda}$ that is marginally consistent with $\mathrm{SN} 1997 \mathrm{ff}$ is $\Omega_{\Lambda}=0.85$ (at the $\sim 3 \sigma$ confidence level), for which the transition redshift occurs at $z=1.25$, which is significantly below the redshift

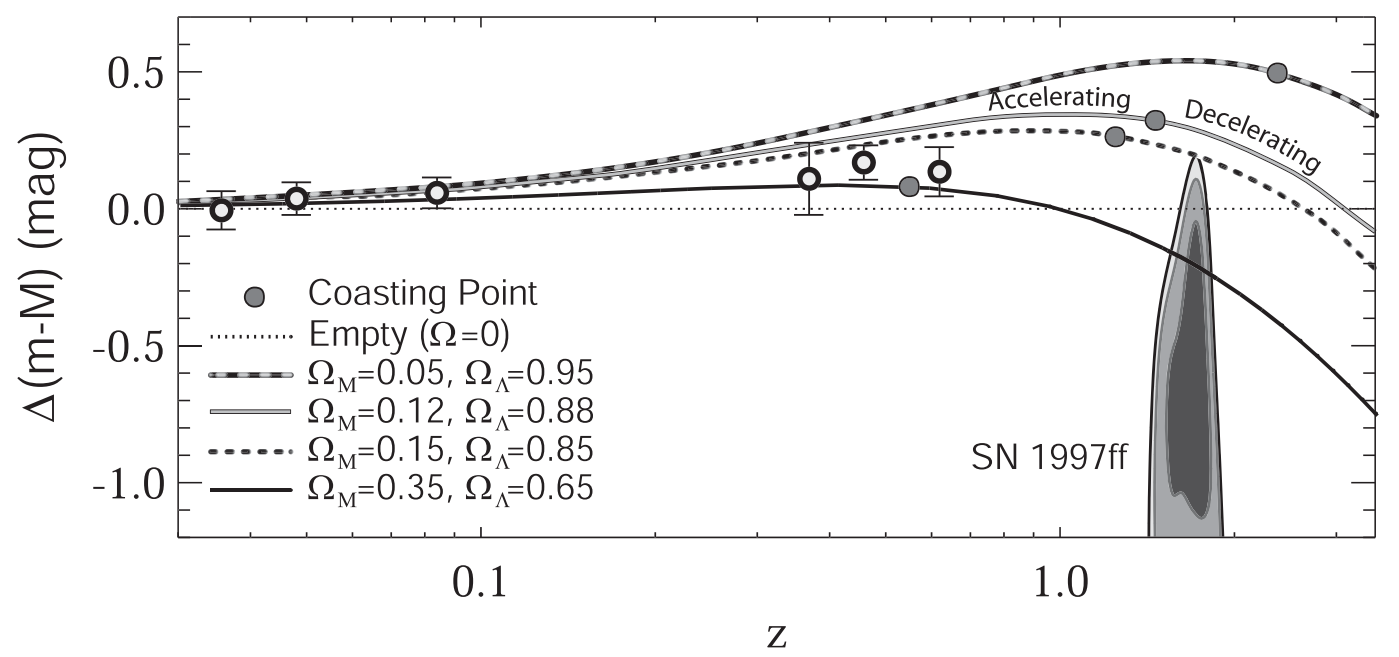

Fig. 12.- Same as Fig. 11 with the inclusion of a family of plausible, flat $\Omega_{\Lambda}$ cosmologies. The transition redshift (i.e., the coasting point) between the accelerating and decelerating phases is indicated and is given as $\left[2 \Omega_{\Lambda} / \Omega_{M}\right]^{1 / 3}-1$. SN $1997 \mathrm{ff}$ is seen to lie within the epoch of deceleration. This conclusion is drawn from the result that the apparent brightness of SN $1997 \mathrm{ff}$ is inconsistent with values of $\Omega_{\Lambda} \geq 0.9$ and, hence, a transition redshift greater than that of SN 1997ff. [See the electronic edition of the Journal for a color version of this figure.] 
of SN 1997ff. For the universe to have commenced accelerating before the explosion of SN $1997 \mathrm{ff}$ requires a value of $\Omega_{\Lambda}>0.9$, a result that is highly in conflict with the $\mathrm{SN}$ brightness. We conclude that, within the framework of these simple but plausible cosmological models, SN 1997ff exploded when the universe was still decelerating. Indeed, the increase in the measured luminosity distance of SNe Ia between $z \approx 0.5$ and $z \approx 1.7$, a factor of 4.0 , is significantly smaller than in most eternally coasting cosmologies (e.g., $\Omega_{M}=0, \Omega_{\Lambda}=0$ ) and appears to favor the empirical reality of a net deceleration over this range in redshift. However, a rigorous and quantitative test of past deceleration requires a more complete consideration of the possible nature of dark energy and is beyond the scope of this paper.

The above conclusions are unchanged if we adopt the tentative spectroscopic redshift of the SN host in place of the photometric redshift indicators [in Figs. 11 and 12, the contours would be replaced by a point at $z=1.755$, $\Delta(m-M)=-0.74 \pm 0.34]$. In this case, the redshift uncertainty is greatly diminished and the distance uncertainty is mildly reduced. However, the dominant source of statistical uncertainty in the testing of this cosmological hypothesis remains the distance uncertainty, not the redshift uncertainty.

A great deal of theoretical effort has been expended recently to understand the nature of dark energy. Some of the possibilities include Einstein's cosmological constant, a decaying scalar field ("quintessence"; Peebles \& Ratra 1988; Caldwell et al. 1998), and so on. A large sample of $\mathrm{SNe}$ Ia distributed over the redshift interval of $0.5<z<2.0$ could empirically break degeneracies between these models (by distinguishing among different average equations of state, $w=P / \rho c^{2}$, where $P$ is the pressure and $\rho$ is the density) if theory alone is insufficient to explain dark energy. SN $1997 \mathrm{ff}$ is in the right redshift range to discriminate between different dark-energy models, and if one assumes that high-redshift $\mathrm{SNe}$ are tracing the cosmological model and not an astrophysical effect, then SN $1997 \mathrm{ff}$ may be useful for this task. However, the large uncertainty present in the measurement of only one SN Ia provides very little leverage to discriminate between dark-energy models at this time.

\section{DISCUSSION}

The results of $\S 3$ indicate that SN $1997 \mathrm{ff}$ is the most distant SN Ia observed to date with a redshift of $z=$ $1.7_{-0.15}^{+0.1}$. Moreover, an estimate of its luminosity distance is consistent with an earlier epoch of deceleration and is inconsistent with astrophysical challenges (e.g., simple evolution or gray dust) to the inference of a currently accelerating universe from $\mathrm{SNe}$ Ia at $z \approx 0.5$. In this section, we explore systematic uncertainties in these conclusions.

\subsection{Supernovae Classification}

$\mathrm{SNe}$ are generally classified by the presence or absence of characteristic features in their spectra. For example, $\mathrm{SNe}$ Ia are distinguished by the absence of hydrogen lines and the presence of Si II $\lambda 6150$ absorption (see Filippenko 1997 for a review). Unfortunately, our inability to observe the defining regions of an SN SED at high redshift necessitates the use of additional indicators of SN type.

However, an alternate way to discriminate some $\mathrm{SNe} \mathrm{Ia}$ is from the morphology of their host galaxies and their associated star formation histories. While all types of SNe have been observed in late-type galaxies, $\mathrm{SNe}$ Ia are the only type to have been observed in early-type galaxies. Although this lore is well known by experienced observers of $\mathrm{SNe}$, this correlation is empirically apparent from an update of the Asiago Supernova Catalog (Cappellaro et al. 1997; Asiago Web site ${ }^{15}$ ) and can be seen in Figure 13. Of the more than $1000 \mathrm{SNe}$ for which type and host galaxy morphologies are all well-defined and have been classified in the modern scheme, there have been no core-collapse $\mathrm{SNe}$ observed in early-type galaxies (that is, only $\mathrm{SNe}$ Ia have been found in such galaxies). All $\sim 40 \mathrm{SNe}$ in elliptical hosts, and classified since the identification of the SN Ia subtype, have been SNe Ia. The same homogeneity of type is true for the $\sim 40 \mathrm{SNe}$ classified in S0 hosts. Core-collapse $\mathrm{SNe}$ (types II, Ib, and Ic) first appear along the Hubble sequence in Sa galaxies, and even within these hosts, they form a minority and their relative frequency to $\mathrm{SNe} I a$ is suppressed by a factor of $\sim 6$ compared to their presence in late-type spirals (Cappellaro et al. 1997, 1999). Evolved systems lose their ability to produce core-collapse SNe.

The explanation for this well-known observation is deeply rooted in the nature of supernova progenitors and their ages. Unlike all other types of $\mathrm{SNe}$ that result from core collapse in massive stars, $\mathrm{SNe}$ Ia are believed to arise from the thermonuclear disruption of a white dwarf near the Chandrasekhar limit and thus occur in evolved stellar populations (see Livio 2000 for a review). The loss of massive stars in elliptical and S0 galaxies, without comparable replacement, quenches the production of core-collapse $\mathrm{SNe}$, while $\mathrm{SNe} I a$, arising from relatively old progenitors, persist. From the host type of SN 1997ff, we might readily conclude, as did GNP99, that it is of Type Ia.

However, more careful consideration is needed to classify SN 1997ff. Because of its high redshift, we need to determine

\footnotetext{
15 The Asiago Supernova Catalog is located at http://merlino.pd.astro.it/ supern/.
}

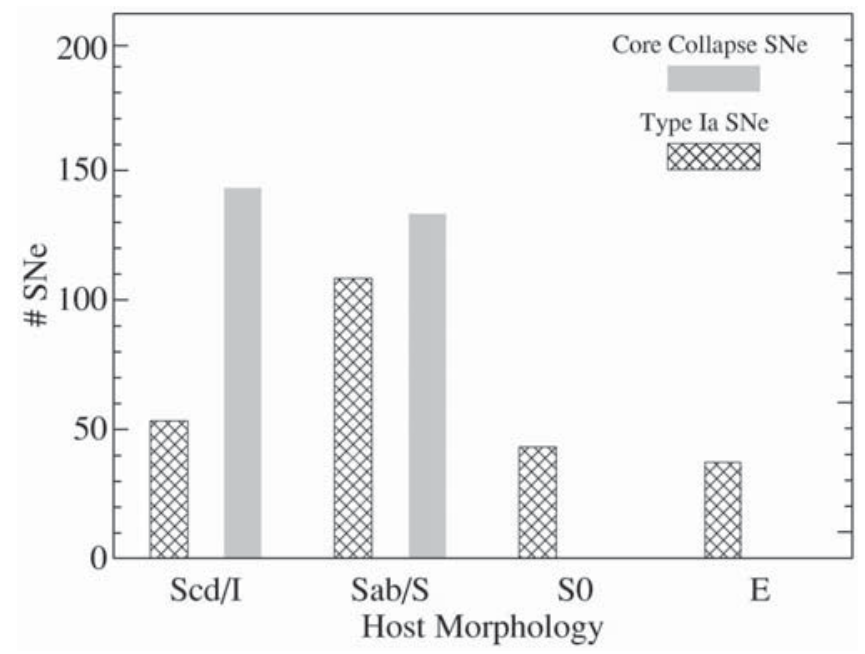

FIG. 13.- SN type vs. host morphology as compiled by the Asiago catalog (Cappellaro et al. 1997). This set includes all SNe through SN 2001X for which a modern SN classification and galaxy classification are available. 
the degree of ongoing star formation and hence the likelihood of the appearance of a core-collapse SN from a young, massive star. While the question of how and when elliptical galaxies form and evolve is beyond the scope of this paper, we are concerned only with the nature of the star formation history and stellar populations in the host of SN $1997 \mathrm{ff}$.

One study of the host galaxy was made previously by Dickinson (1999; see also M. Dickinson et al. 2001, in preparation), who used IR NICMOS photometry to compare the rest-frame $B-V$ colors of high-redshift HDF-N ellipticals to those expected for different formation and evolution scenarios. Dickinson found the host and another nearby red elliptical to have rest-frame $B-V$ colors consistent with a burst of star formation at $z \approx 4$ or 5 followed by passive evolution.

Here we extend this analysis by comparing the complete ultraviolet-optical-infrared SED of the host galaxy to Bruzual \& Charlot (1993) population synthesis models. The upper panel of Figure 14 superimposes the galaxy photometry with models that assume a single, short burst of star

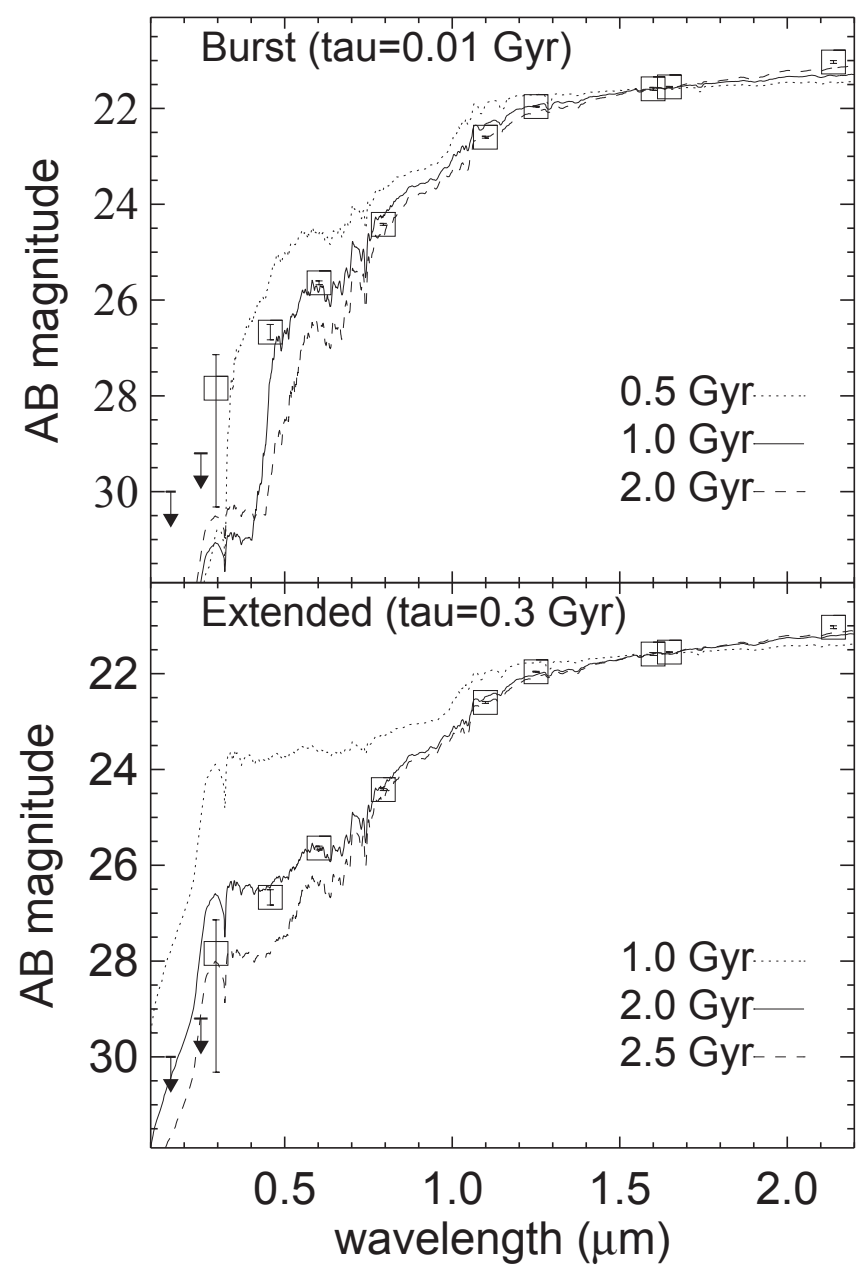

FIG. 14.-Possible star formation histories of the host of SN 1997ff compared to its observed SED. The upper panel shows the expected SED for a single burst of star formation occurring at $0.5 \mathrm{Gyr}, 1 \mathrm{Gyr}$, and $2 \mathrm{Gyr}$ before the explosion SN 1997ff. The favored age of $1 \mathrm{Gyr}$ is long after the loss of the progenitors of core-collapse SNe. For a star formation history consisting of a burst (lower panel) followed by continuous and exponential decay of extended star formation $(\tau=0.3 \mathrm{Gyr})$, the expected ratio of $\mathrm{SNe}$ Ia to core-collapse $\mathrm{SNe}$ is between 20 and 70 . formation with a Salpeter initial mass function. Such a model has negligible ongoing star formation after the initial burst, and thus its rest-frame ultraviolet (UV) to optical colors redden as quickly as possible. After 1 Gyr has elapsed, this model approximately matches the observedframe colors of the host galaxy; a burst occurring 0.5 or 2.0 Gyr before the SN appears too short and too long, respectively. We expect very few remaining massive stars $\geq 1 \mathrm{Gyr}$ after the cessation of star formation and, therefore, a negligible chance that SN 1997ff could be a core-collapse SN (the progenitors of which live for less than $40 \mathrm{Myr}$ ). An alternative history would extend the star formation timescale, providing a small residual of ongoing star formation to boost the UV flux while allowing the rest-frame optical colors to redden to match the $I J H K$ photometry. The bottom panel of Figure 14 shows such a model, with an exponential star formation timescale of $0.3 \mathrm{Gyr}$. This model matches the observed SED at an age between 2.0 and $2.5 \mathrm{Gyr}$ (with the far-UV limit favoring the older age). Normalized to the $H$-band magnitude of the galaxy, and assuming $\Omega_{M}=0.3$, $\Omega_{\Lambda}=0.7$, and $H_{0}=70 \mathrm{~km} \mathrm{~s}^{-1} \mathrm{Mpc}^{-1}$ to compute the luminosity distance, this model provides an ongoing star formation rate of 0.7 to $0.2 M_{\odot} \mathrm{yr}^{-1}$ at the time SN $1997 \mathrm{ff}$ exploded $\left[(2.4-10) \times 10^{-4}\right.$ times the initial rate $]$. The remaining population of massive stars should produce 0.004 to 0.001 core-collapse $\mathrm{SNe}$ per year (in the rest frame). We employ a more empirical route to determine the expected rate of $\mathrm{SNe}$ Ia caused by our inability to identify conclusively their progenitor systems. Estimates for the rate of $\mathrm{SNe}$ Ia at high redshift from Pain et al. (1996) yield 0.48 SNe Ia per century per $10^{10}$ solar blue luminosities $\left(H_{0}=70 \mathrm{~km}\right.$ $\mathrm{s}^{-1} \mathrm{Mpc}^{-1}$ ). Sullivan et al. (2000) and Kobayashi, Tsujimoto, \& Nomoto (2000) predict a rise in this rate by a factor of $\sim 2$ at the redshift of SN 1997ff. The host galaxy luminosity is $M_{B}=-21.9$; hence, we expect a rate of $\sim 0.07$ $\mathrm{SNe}$ Ia per year. We thus expect the host to produce 20 to 70 times as many $\mathrm{SNe}$ Ia as core-collapse $\mathrm{SNe}$ at the time SN 1997ff exploded (with the far-UV limit favoring the larger ratio), favoring its classification as an SN Ia independent of the cosmological model.

A longer timescale of star formation pushes the time of the initial burst uncomfortably close to the formation of globular clusters without significantly altering the expected production ratio of core-collapse $\mathrm{SNe}$ to $\mathrm{SNe} \mathrm{Ia}$.

The very tentative identification of a noisy spectral feature with [O II] emission would provide an [O II] flux $f([\mathrm{O}$ II $]) \approx 8.7 \times 10^{-18}$ ergs s $^{-1}$, with a very large uncertainty (at least 50\%) because of its low $\mathrm{S} / \mathrm{N}$. As discussed by Kennicutt (1998), the conversion from [O II] line flux to star formation rate is imprecise, and large variations in [O II] $/ \mathrm{H} \alpha$ (as much as $0.5-1 \mathrm{dex}$ ) are seen among local galaxies. Nevertheless, adopting Kennicutt's conversion for a Salpeter initial mass function (IMF), and assuming (as in $\S 4.1$ ) a cosmology with $\Omega_{M}=0.3, \Omega_{\Lambda}=0.7$, and $H_{0}=70 \mathrm{~km}$ $\mathrm{s}^{-1} \mathrm{Mpc}^{-1}$, we estimate a host galaxy star formation rate of 2.6 $M_{\odot} \mathrm{yr}^{-1}$ from the tentative [O $\left.\mathrm{II}\right]$ line identification. This is 4 to 13 times larger than the rates we estimated from the broadband photometric modeling and would result in a rate of core-collapse $\mathrm{SNe}$ of $\sim 0.01 \mathrm{yr}^{-1}$, still a factor of $\sim 10$ smaller than our estimate of the Type Ia $\mathrm{SN}$ rate. However, we consider the identification of this spectral feature as $[\mathrm{O}$ II $]$ emission very tentative and the putative flux very uncertain; therefore, the calculation of its implied star formation rate is highly speculative. 
Another route to estimating the fraction of core-collapse progenitors comes from a direct conversion of rest-frame UV flux (e.g., from the $B_{450}$ magnitude, which corresponds to roughly $1650 \AA$ in the host-galaxy rest frame at $z=1.7$ ) to star formation rates, following the conversion for a Salpeter IMF from Kennicutt (1998). This yields an estimated star formation rate of $0.8 M_{\odot} \mathrm{yr}^{-1}$, which is consistent with the results derived previously using an extended star formation scenario. However, this value is likely to be an overestimate since the standard conversion factor is estimated from models with constant star formation rates while the galaxy colors resemble those of an early-type galaxy for which the current star formation rate is almost certainly far lower than its past average. Some significant fraction of the UV light may therefore come from longer lived stars and less need be attributed to ongoing star formation.

A potentially powerful tool to discriminate between $\mathrm{SN}$ types comes from enlisting the observed SN data set. Both the High-z Supernova Search Team and the Supernova Cosmology Project have relied on the photometric behavior of an SN when a useful spectrum was not available (Riess et al. 1998; Perlmutter et al. 1999). The distanceindependent observables of color and light-curve shape have the potential to discriminate Type Ia SNe from other SN types. As discussed in $\S 3.1$, from the observed colors and decline of SN 1997ff, we conclude that its photometric behavior is inconsistent with a Type II supernova at any redshift. Also, the scarcity of SNe IIn having similar photometric properties argues against SN 1997ff being an SN IIn on photometric grounds. In contrast, the goodness-of-fit between SN $1997 \mathrm{ff}$ and an empirical model of an SN Ia at $z=1.7$ discovered a week after maximum and with a typical light-curve shape (see the middle panel of Fig. 7) is highly consistent with the SN Ia identification (reduced $\chi^{2}=0.5$ for $\sim 10$ degrees of freedom). Coupled with the apparent consistency of the two photometric redshifts and the tentative spectroscopic redshift, we might consider the $\mathrm{SN}$ data to be the best arbiter of type.

Unfortunately, when using only photometric information, it may be possible to confuse an SN Ia with a luminous SN Ic or SN Ib (Clocchiatti et al. 2000; Riess et al. 1998). However, $\mathrm{SNe} \mathrm{Ib}$ and Ic are far rarer than $\mathrm{SNe}$ Ia (Cappellaro et al. 1997, 1999), and they are expected to arise from even more massive progenitors than SNe II (e.g., WolfRayet stars); if so, these progenitors should be even less populous than those of SNe II in the comparatively red host of SN 1997ff. (However, the masses of progenitors of $\mathrm{SNe} \mathrm{II}$ and $\mathrm{SNe} \mathrm{Ib} / \mathrm{Ic}$ can overlap, if the hydrogen envelope of the progenitor can be lost through mass transfer in a binary system; e.g., Filippenko 1997) Empirically, SNe Ib and Ic are common only in very late-type galaxies (i.e., Sc; Cappellaro et al. 1997, 1999), environments of marked contrast to the host of SN 1997ff. The rare, peculiar, highly luminous supernovae ("hypernovae") that may be associated with gamma-ray bursts, such as SN 1998bw (e.g., Galama et al. 1998; Iwamoto et al. 1998; Woosley, Eastman, \& Schmidt 1999), SN 1997cy (Germany et al. 2000; Turatto et al. 2000), and SN 1999E (Filippenko 2000), also seem to be produced by core collapse in very massive stars.

Based on the nature of the host galaxy (an evolved, red elliptical) and diagnostics available from the observed colors and temporal behavior of the $\mathrm{SN}$, we find the most likely interpretation is that SN $1997 \mathrm{ff}$ was of Type Ia.

\subsection{Caveats}

Because we have employed the SN colors to seek constraints on the SN redshift and age at discovery, both of which are strong functions of SN color, we cannot employ the colors to determine if there is any reddening by interstellar dust. The HDF-N was chosen (at high Galactic latitude) in part to minimize foreground extinction, so we assume that Milky Way reddening of SN 1997ff is negligible. Similarly, given the evolved nature of the red, elliptical host, we assume negligible reddening by the host of the SN. However, the apparent consistency with past cosmological deceleration and the apparent inconsistency with contaminating astrophysical effects reported here would not be challenged by unexpected, interstellar reddening to $\mathrm{SN}$ 1997 ff. To demonstrate this conclusion, we reddened the SN by $A_{B}=0.25 \mathrm{mag}$ in the rest frame and recalculated the PDF in the distance-redshift plane. As shown in Figure 11, the fit is shifted along a "reddening vector" farther away from the model of astrophysical effects or cosmological nondeceleration. While we consider solutions along the reddening vector less likely, they are important to bear in mind when assessing quantitative estimates of cosmological parameters based on the previous analysis.

Our empirical model of evolution or intergalactic gray extinction (in magnitudes) is highly simplistic (i.e., linear) and consists only of the product of a constant and the redshift. Relative to the empty cosmology $\left(\Omega_{M}=0, \Omega_{\Lambda}=0\right)$, this constant is chosen to be $0.3 \mathrm{mag}$ per unit redshift to match the observed distances of SNe Ia at $z \approx 0.5$. The functional form of this model is the same as that derived by T. York et al. (2001, in preparation) from the consideration of a dust-filled universe and is shown to be valid for redshifts near unity. It also approximates the calculations of Aguirre (1999a, 1999b). However, depending on the epoch when the hypothetical dust is expected to form, the optical depth might be expected to drop at a redshift higher than two.

Evolution is far more difficult to model and predict (Höflich et al. 1998; Umeda et al. 1999a, 1999b; Livio 2000). For this hypothetical astrophysical effect, our model is that the amount of luminosity evolution would scale with the mean age available for the growth of the progenitor system (for $z \approx 1$ ). While more complex parameterizations are possible, the salient feature of our simple luminosity evolution model is its monotonic increase with redshift.

Drell et al. (2000) considered somewhat more complex phenomenological models of evolution (in magnitudes) consisting of a variable offset and a variable coefficient multiplied by the logarithm of $(1+z)$. While the functional form of this model may be less motivated by considerations of the natural scaling of the physical parameters involved (e.g., time or metallicity), the additional free parameters make it possible to empirically fit both the observed dimming $(z \approx 0.5)$ and the observed brightening $(z \approx 1.7)$ within a wider range of underlying cosmological models (e.g., Einstein-de Sitter). To test higher order parameterizations of evolution than the one we considered here will require measurements of more distant $\mathrm{SNe}$ Ia in new redshift intervals.

As discussed in Appendices A and B, constraints derived from the photometry of SN 1997ff can be recovered from any of the methods used to characterize the relationship between SN Ia light curves, color curves, and luminosity. 
To test the sensitivity of our analysis to the light-curve shape method employed, we rederived the SN constraints in $\S 3$ using the "stretch method" described by Perlmutter et al. (1997). The results were in excellent agreement with those presented using the MLCS method (Riess et al. 1998) in $\S 3$. The only noteworthy exception is that the constraints on the expected luminosity and distance of SN 1997ff were somewhat narrower for the stretch-method analysis. The explanation for this difference can be found in the calibration of the relationship between the peak luminosity and light-curve shape used by each method. The stretch method expects a somewhat smaller change in peak luminosity for a given variation in light-curve shape than the MLCS method. For the range of possible light-curve shapes allowed by the quality of the fit to the SN $1997 \mathrm{ff}$ data, the stretch method therefore predicts a smaller variation in luminosity (and hence distance) for SN 1997ff than the MLCS method. The cosmological conclusions reported here are supported by either method.

Sample selection biases can be important factors to consider when employing sources detected in a magnitudelimited survey (e.g., Li et al. 2001). However, because cosmological measurements from $\mathrm{SNe}$ Ia are generally based on the difference in the apparent luminosities of $\mathrm{SNe}$ from such surveys, a propagated bias in the cosmological measurements is greatly diminished (Schmidt et al. 1998; Riess et al. 1998; Perlmutter et al. 1999). In addition, the relatively low intrinsic scatter of SN Ia distance measurements $(\sigma=0.15 \mathrm{mag})$ further reduces such biases. Monte Carlo simulations of these biases indicate that the appropriate corrections to the measured distance moduli are less than 0.05 mag, which is negligible compared with the statistical uncertainties for SN $1997 \mathrm{ff}$ presented here.

It is important to devote special consideration to the likelihood that SN 1997ff resembles the overluminous and slow declining SN 1991 T (Filippenko et al. 1992a). An unexpected overluminosity of SN $1997 \mathrm{ff}$ would result in an overestimation of the apparent disagreement with an astrophysical source of dimming. The best estimate of the overluminosity of SN $1991 \mathrm{~T}$ is $0.3 \pm 0.3 \mathrm{mag}$ based on a recent determination of the Cepheid distance to the host using HST (Saha et al. 2001). If SN 1997ff were unexpectedly overluminous by this amount, it would still remain brighter than expected for the dust or simple evolution model (by about $0.8 \mathrm{mag}$ ), but the significance of the difference would be reduced. However, the possibility that SN $1997 f f$ matches SN 1991T-like SNe is explicitly included in the analysis in $\S 3$ by comparing the fit between the photometry of the former and the latter (and by employing the widest luminosity prior). The good fit between SN 1997ff and a typical SN Ia disfavors its identification with the slower declining SN 1991T. Empirically, SN 1991T-like events appear to favor hosts with younger stellar populations (Hamuy et al. 2000; Howell 2000), but this diagnostic is not as useful as the observed light curve shape in determining the likelihood that SN 1997ff resembles SN $1991 \mathrm{~T}$.

Clustering of mass in the universe can cause the line of sight to most SNe to be underdense relative to the mean, while an occasional supernova may be seen through an overdense region. In Riess et al. (1998) and Perlmutter et al. (1999), stochastic lensing that decreased typical fluxes caused by underdense lines of sight to the SNe was considered and found to have little effect on the cosmological measurements. The typical deamplification would be larger at $z \approx 1.7$ and may approach a $10 \%-15 \%$ decrease in the observed brightness of a typical SN such as SN 1997ff (Holz 1998). For a large sample of $\mathrm{SNe}$, the mean would provide an unbiased estimate of the unlensed value, but for a single $\mathrm{SN}$, median statistics are more robust and deamplification of SN 1997ff is applicable.

Lensing by the foreground large-scale structure can also alter the apparent brightness of a distant supernova (Metcalf \& Silk 1999) in the opposite sense of the preceding consideration. There is a pair of galaxies in the foreground of SN 1997ff at $z=0.56$, with a separation of $\sim 3^{\prime \prime}$ and 5.5 from the SN. If we assume $H_{0}=70 \mathrm{~km} \mathrm{~s}^{-1} \mathrm{Mpc}^{-1}, \Omega_{M}=$ 0.3 , and $\Omega_{\Lambda}=0.7$, these galaxies are found at projected distances of $\sim 20$ and $35 \mathrm{kpc}$ in the lens plane and have approximately $L^{*}$ luminosity, with $M_{V} \approx-21$, implying a velocity dispersion of $\sim 200 \mathrm{~km} \mathrm{~s}^{-1}$. Assuming that the two galaxies have an approximately isothermal mass distribution, the resulting magnification of SN $1997 \mathrm{ff}$ would be $\sim 0.3 \mathrm{mag}$, in good agreement with the results of Lewis \& Ibata (2001). However, without detailed knowledge of the form of the mass potential, it is not possible to recover the precise amplification (and to accurately correct the measured luminosity). Our estimate is only an approximation since the mass profile could fall off more steeply than assumed, or there could be an excess dark matter concentration associated with this pair of galaxies.

The $\mathrm{H} \alpha$ line width measured from the NIRSPEC spectrum for the nearby $z=0.56$ foreground galaxy is not significantly resolved at an instrumental resolution of about $180 \mathrm{~km} \mathrm{~s}^{-1}$ (FWHM), indicating $\sigma \leq 90 \mathrm{~km} \mathrm{~s}^{-1}$ with no indication of any rotational sheer. This may not provide a strong constraint on the foreground galaxy mass, given uncertainties in the galaxy orientation (it appears to be somewhat face-on, although this is difficult to assess given its irregular morphology) and the fact that the line emission may trace only one star-forming region within the galaxy rather than the full potential well depth. We can only say that there is no immediate kinematic evidence from existing spectroscopy for a large mass for the closest foreground galaxy.

It is possible to derive a useful constraint on the maximum likely amplification of the $\mathrm{SN}$ by the closest foreground lenses by examining the shape of the host galaxy that would be stretched in the tangential direction by an amount that depends on the SN amplification. This calculation is performed in Appendix $\mathrm{C}$ and the results can be seen in Figure 15. From this calculation, we conclude that the lack of apparent tangential ellipticity of the host galaxy (for the degree of specific $\mathrm{SN}$ amplification estimated above) is not very surprising $(\sim 20 \%$ of randomly selected hosts would exhibit as little tangential stretching as seen). However, significantly greater amplifications are not very likely; 0.6 or 0.8 mag amplifications would produce galaxies with no evidence of tangential stretching only in $6 \%$ and $3 \%$ of identical ensembles, respectively. The observed roundness of the image of the host galaxy, with an axis ratio of 0.85 , is further circumstantial evidence against the presence of substantial lensing. While unremarkable in the absence of lensing, such roundness is unusual in images produced from highly elliptical galaxies oriented so as to counteract the tangential stretching caused by lensing. We estimate that only $\sim 4 \%$ of randomly selected hosts will look as round as observed in the presence of $0.4 \mathrm{mag}$ of amplification, and the fraction 


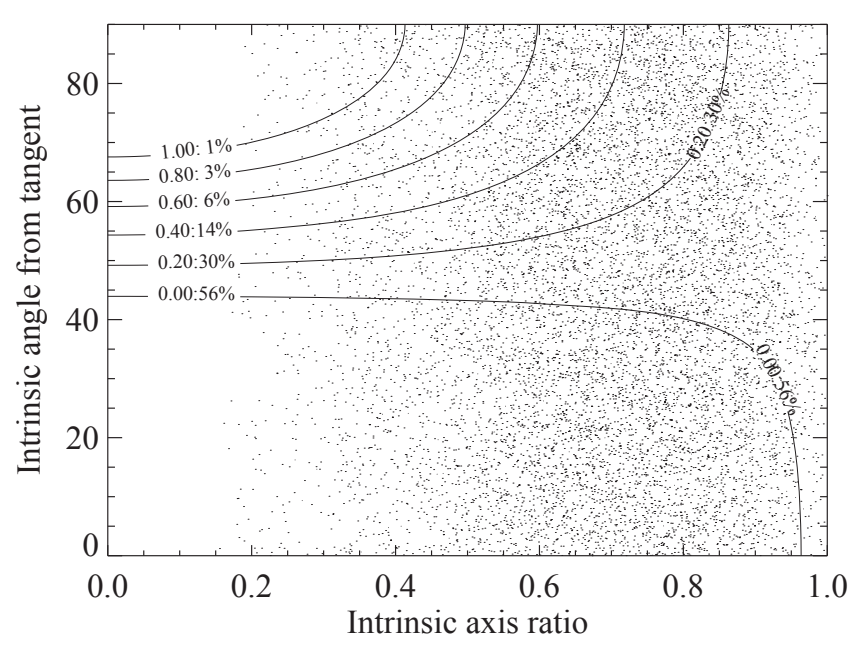

FIG. 15.-Tangential ellipticity test for the SN host galaxy. The points show a subset $\left(10^{4}\right.$ of the $10^{6}$ samples $)$ of randomly selected combinations of the intrinsic axis ratio and orientation angle of simulated host galaxies. For several different hypothetical values of the $\mathrm{SN}$ amplification, a curve is shown indicating the combinations of intrinsic axis ratio and orientation angle resulting in the observed tangential eccentricity of the SN host. Each curve is labeled with a hypothetical amplification (in magnitudes) of the $\mathrm{SN}$ and the fraction of randomly selected galaxies for which observed tangential ellipticity would be as small as that observed or smaller (see Appendix C).

drops below $1 \%$ for a magnification of $0.8 \mathrm{mag}$. However, this test is more circumstantial and therefore less preferable than the tangential ellipticity test described in Appendix C.

Together, the specific and stochastic lensing cases result in a possible net $\sim 0.2 \mathrm{mag}$ shift in the luminosity. This value is considerably smaller than the $\sim 1$ mag observed difference between the SN luminosity and that expected for astrophysical contaminants (gray dust or evolution), although it is in the direction to reduce this spread. Thus, our cosmological conclusions appear robust to lensing effects, although we cannot rule out more exotic lensing scenarios (e.g., a massive dark matter sheet amplifying the $\mathrm{SN}$ but not shearing the host). Moreover, given that a $\sim 0.2$ mag error caused by lensing remains a potential source of systematic uncertainty, we discourage future attempts to refine the distance measurement to SN $1997 \mathrm{ff}$ without a careful consideration of the full impact of weak lensing. The challenge posed by disentangling the affects of lensing and apparent distance provide a strong impetus to collect more $\mathrm{SNe}$ Ia at $z>1$ to reduce the statistical impact of such degeneracies.

We note that the reported consistency with cosmological deceleration and the exclusion of the dust model or a simple evolution model for SN 1997ff would remain unchanged in light of a future, high-precision measurement of the redshift of the host if it is found to be within the redshift interval $1.4<z<1.95$. This interval is the range over which the SN data are even minimally consistent with the empirical models of SNe Ia. A future redshift determination that is inconsistent with this range would cast serious doubt on the interpretation of SN 1997ff as a familiar SN Ia or any conclusions based on this interpretation.

Finally, the detection of SNe Ia at high redshifts can add potentially valuable insights into the nature of the progenitors of SNe Ia. Unfortunately, with the single case of SN 1997ff, the conclusions are not very restrictive, and perhaps even circular, since the fact that it occurred in an elliptical galaxy was used to infer that it is an $\mathrm{SN}$ Ia; many $\mathrm{SNe}$ Ia may be needed, preferably with spectroscopic confirmation, over a range of redshifts to constrain the progenitors of $\mathrm{SNe}$ Ia. The time allowed for the progenitor system of SN $1997 \mathrm{ff}$ to form and evolve is likely to be more than $1 \mathrm{Gyr}$ (based on the star formation history of the elliptical host) and less than about 4 Gyr (based on the time interval between the redshift of the SN and that of the initial formation of stars). However, we already know that the progenitors of some SNe Ia are very old $\left(\sim 10^{10} \mathrm{yr}\right)$, since they are found in present-day, gas-deficient, old elliptical galaxies. Also, it already appears that the progenitors of most $\mathrm{SNe}$ Ia are relatively young ( $\lesssim 1 \mathrm{Gyr}$ ), since they are found with greatest frequency in late-type galaxies (Cappellaro et al. 1997, 1999) and might even exhibit a loose correlation with spiral arms (Bartunov, Tsvetkov, \& Filimonova 1994).

\section{CONCLUSIONS}

1. SN 1997ff is the highest redshift $\mathrm{SN}$ Ia observed to date, and we estimate its redshift to be $\sim 1.7$. This redshift is consistent with the measurements made from either the SN data, the nine-band photometric redshift of the host, or the tentative indication from the host-galaxy spectroscopy. The classification as an SN Ia is derived from observational and theoretical evidence that the evolved, elliptical host is deficient in the progenitors of core-collapse SNe. The classification is also supported by diagnostics available from the observed colors and temporal behavior of the $\mathrm{SN}$.

2. The derived constraints for the redshift and distance of SN $1997 \mathrm{ff}$ are consistent with the early decelerating phase of a currently accelerating universe and thus are a valuable test of a universe with dark energy. The results are inconsistent with simple evolution or gray dust, the two most favored astrophysical effects which could mimic previous evidence for an accelerating universe from $\mathrm{SNe}$ Ia at $z \approx 0.5$.

3. We consider several sources of potential systematic error, including gravitational lensing, supernova misclassification, sample selection bias, and luminosity calibration errors. Currently, none of these effects alone appears likely to challenge our conclusions. However, observations of more $\mathrm{SNe}$ Ia at $z>1$ are needed to test more complex challenges to the accelerating universe hypothesis and to probe the nature of dark energy.

We wish to thank Chris Fassnacht, Kailash Sahu, Nick Suntzeff, Bruno Leibundgut, Steve Beckwith, Sean Carroll, Harry Ferguson, Greg Aldering, and Casey Papovich for valuable contributions and discussions. We are indebted to Michael Turner for his efforts to refine our consideration of a past deceleration. Parts of this project were supported by grants GO-6473, GO-7817, and AR-7984 from the Space Telescope Science Institute, which is operated by the Association of Universities for Research in Astronomy, Inc., under NASA contract NAS 5-26555; we also acknowledge NASA support of the NICMOS GTO team. A. V. F. is grateful for a Guggenheim Foundation Fellowship and for NSF grant AST-9987438. This work was supported by a NASA LTSA grant to P. E. N. and by the Director, Office of Science under US Department of Energy Contract No. DE-AC03-76SF00098. P. E. N. also thanks NERSC for a generous allocation of computer time. 


\section{APPENDIX A}

\section{SUPERNOVA AND COSMOLOGICAL LIKELIHOOD FUNCTION}

Our goal is to define a simple formalism to combine the popular light-curve fitting methods with prior information (e.g., a likelihood function) in a statistically sound way. The motivation for this method is for application to sparse or inhomogeneous SN Ia data sets that can be augmented with additional information. Current light-curve fitting methods can include four free parameters (age, distance, extinction, and luminosity) and could include more, such as a photometric redshift or an extinction law that can be constrained from SN colors. Alternatively, it may be desirable to discard the two parameters of distance and redshift in exchange for cosmological parameters such as $H_{0}, \Omega_{m}, \Omega_{\Lambda}, w$, etc. However, data sets gathered at high redshift are often noisy, sparse, and inhomogeneous, and may provide a high or low degree of leverage on any of these parameters. It may be desirous to supplement the data set with prior information, such as a host galaxy photometric redshift or a luminosity function, while adding no additional information to some parameters, such as the age at discovery.

Of course, a powerful language for such formalism is Bayes's theorem. In our case,

$$
p(\boldsymbol{q} \mid \boldsymbol{D})=\frac{p(\boldsymbol{D} \mid \boldsymbol{q}) p(\boldsymbol{q})}{p(\boldsymbol{D})},
$$

where $\boldsymbol{D}$ is a set of SN data and priors, and $\boldsymbol{q}$ is a combination of SN or cosmological parameters we are trying to constrain. Because we have no prior constraints on $D$, we have

$$
p(\boldsymbol{q} \mid \boldsymbol{D}) \propto p(\boldsymbol{D} \mid \boldsymbol{q}) p(\boldsymbol{q}) .
$$

In principle, $\boldsymbol{q}$ should contain the set of all SN or cosmological parameters that cause our data to appear as they do, but in practice it is necessary only to include parameters to which our data set is sensitive. Here we consider the set of parameters $\mu_{B}$ (apparent distance modulus), $t_{d}$ (days past $B$-band maximum at the time of discovery), $\Delta$ (the MLCS luminosity/light-curveshape parameter; Riess et al. 1996), and $z$ (the redshift). Also, $\boldsymbol{D}$ is the set $\boldsymbol{m}$ of magnitude measurements in Table 1 for SN 1997ff.

As a result, we have

$$
p\left(\mu_{B}, t_{d}, \Delta, z \mid \boldsymbol{m}\right) \propto\left[\prod_{i}\left(\frac{1}{\sqrt{2 \pi \sigma_{m, i}^{2}}}\right)\right] \exp \left(-\frac{\chi^{2}}{2}\right)\left[p\left(t_{d}\right) p\left(\Delta \mid t_{d}\right) p\left(\mu_{B} \mid t_{d}, \Delta\right) p\left(z \mid \mu_{B}, t_{d}, \Delta\right)\right],
$$

and $\chi^{2}$ is defined as

$$
\chi^{2}\left(\mu_{B}, t_{d}, \Delta, z\right)=\sum_{i} \frac{\left[\mu_{B}+M_{B}\left(\Delta, t_{d}, t_{i}, z\right)-m_{i}+K_{i}\left(z, t_{i}, t_{d}, \Delta\right)\right]^{2}}{\sigma_{m_{i}}^{2}} .
$$

The term $M_{B}\left(\Delta, t_{d}, t_{i}, z\right)$ is an empirical light-curve model of an SN Ia in the $B$ band. To compare an apparent magnitude measurement $m_{i}$ at time $t_{i}$, we compute the expected magnitude of an SN MLCS model $M_{\boldsymbol{B}}(\Delta)$ at the rest-frame age relative to $B$-band maximum of $\left(t_{i}-t_{d}\right) /(1+z)$. The term $K_{i}\left(z, t_{i}, t_{d}, \Delta\right)$ is a cross-filter $K$-correction and is discussed extensively for $\mathrm{SNe}$ Ia elsewhere (Kim, Goobar, \& Perlmutter 1996; Riess et al. 1998; Schmidt et al. 1998; P. E. Nugent, A. Kim, \& S. Perlmutter 2001 , in preparation). It is determined by synthetically calculating the pseudocolor $X-B$ (where $X$ represents the passband F814W, F110W, or F160W blueshifted by $1+z$ ) using the SED of an SN Ia (with intrinsic colors appropriate to an SN Ia with a luminosity parameter of $\Delta)$ at an age relative to $B$ maximum of $\left(t_{i}-t_{d}\right) /(1+z)$.

The PDFs in brackets on the right-hand side of equation (A3) are priors in which we can include any desired amount of prior information about these parameters. $p\left(t_{d}\right)$ is the prior distribution on the age at which the SN was discovered given no other information. A spectrum of an SN Ia can be used to set a prior constraint on the age (Riess et al. 1997), or without such information we would choose this prior to be constant. $p\left(\Delta \mid t_{d}\right)$ is the observed luminosity function of SNe Ia. $p\left(\mu_{B} \mid t_{d}, \Delta\right)$ is given by the area of the hypershell with a radius of $\mu_{B}$. In principle, this prior, derived from the area of hypershells, is cosmology-dependent because the geometry of these hypershells is determined by the cosmological parameters. However, for SN data that are moderately constraining (such as is the case for SN 1997ff), the specific cosmology chosen has a negligible impact on the fits. If this were not the case, it would be necessary to substitute the "nuisance parameters" of distance and redshift for cosmological parameters and the additional constraint in the form of the equation of the luminosity distance (Schmidt et al. 1998). For now treating $z$ and distance as independent, the term $p\left(z \mid \mu_{B}, t_{d}, \Delta\right)$ contains the prior constraint on the redshift (such as a photometric redshift) and possibly a time dilation effect such as the $1+z$ expansion in time intervals (and hence the likelihood of finding an SN Ia at higher redshifts).

Either the $\chi^{2}$ statistic or the a posteriori probability may be used in the usual ways to determine constraints on any combinations of the parameters $q$ (Press et al. 1992).

\section{APPENDIX B}

\section{CROSS-BAND K-CORRECTIONS}

For a full description of the $K$-corrections and the uncertainties involved with their application, see P. E. Nugent, A. Kim, \& S. Perlmutter (2001, in preparation). In what follows, we briefly summarize the relevant issues that concern us here. 
The magnitude of an SN Ia in filter $y$ can be expressed as the sum of its absolute magnitude $M_{x}$, cross-filter $K$-correction $K_{x y}$, distance modulus $\mu$, and extinction caused by dust in both the host galaxy, $A_{x}$, and our galaxy, $A_{y}$ :

$$
m_{y}[t(1+z)]=M_{x}(t, s)+K_{x y}(z, t, s)+\mu\left(z, \Omega_{M}, \Omega_{\Lambda}, H_{o}\right)+A_{x}+A_{y} .
$$

Here $t$ refers to the epoch when the SN Ia is being observed and $z$ is its redshift. The parameter, $s$, can be any parameter (or method) that is used to characterize the relationship between the light curves, colors, and luminosity of an individual SN Ia. For the MLCS method (Riess et al. 1996, 1998), this parameter would be the $\Delta$ parameter, formally the peak absolute luminosity of the visual light curve (relative to a fiducial SN Ia). Alternatively, it could be the $\Delta m_{15}(B)$ parameter $(\mathrm{Phillips}$ 1993; Phillips et al. 1999) or the stretch-factor (as described in Perlmutter et al. 1997).

The spectral template used to produced the $K$-corrections was created by gathering together all the spectra of wellobserved SNe Ia currently available to the authors. Especially important to these calculations were the SNe observed in the UV by IUE and HST (Cappellaro, Turatto, \& Fernley 1995; Kirshner et al. 1993). A set of standard photometric templates was then created for a fiducial SN Ia as well as SNe Ia with values of $s$ chosen to sample and span the observed range of SNe Ia in $U B V R I$. The spectra were then "flux calibrated" in order to reproduce the observed magnitudes of these template light curves by both adjusting the zero point of the flux scale and applying a slope correction to the flux so that each spectrum would have the correct color (Riess et al. 1996, 1998) for a particular phase. The slope correction was performed by altering the flux using the reddening law of Cardelli, Clayton, \& Mathis (1989) (either making them bluer or redder accordingly). For the list of the SNe Ia and their corresponding UBVRI magnitudes, see P. E. Nugent, A. Kim, \& S. Perlmutter (2001, in preparation).

To determine the $K$-correction for a given value of $s$ and $z$, the set of spectra were first flux calibrated (as described above) to match the fluxes of the set of template light curves described by the parameter $s$. The cross-band $K$-correction was then calculated according to the formulae of Kim et al. (1996; see also Schmidt et al. 1998). After identification of the observed bandpass and a rest-frame bandpass, a pseudocolor is numerically calculated. This pseudocolor is the difference in the magnitude of the SN in the rest-frame bandpass and the observed-frame bandpass, the latter blueshifted by $(1+z)$, and is calculated from the template SN spectrophotometry. These calculations are derived from spectrophotometry of SNe Ia at different phases, and we interpolate these values to determine the correction at a given phase. Examples of these calculations are given in Table 2 ; they are uncertain to less than $10 \%$.

\section{APPENDIX C}

\section{THE TANGENTIAL ELLIPTICITY TEST FOR THE SUPERNOVA HOST GALAXY}

Significant gravitational lensing caused by galaxies close to the line of sight would be expected to cause a detectable distortion - primarily a tangential stretch - of the shape of the host galaxy. The observed tangential ellipticity can in turn be used to constrain the amplification caused by lensing. However, the intrinsic shape of the host galaxy is not known, and thus it is possible that the galaxy is intrinsically elongated in the radial direction so that the final image does appear nearly round.

We can, however, apply a statistical test to determine the likelihood that we would be able to detect the tangential stretch. We ask how frequently, for a randomized distribution of the intrinsic (i.e., unlensed) parameters of the galaxy, the lensed image will appear to have as little tangential stretch as is observed. (Note that this test is one-sided since we know that the lens causes a tangential stretch.)

For the sake of simplicity, we first limit our consideration to lensing from the nearest bright galaxy, about $3^{\prime \prime}$ north and 0 ".6 east of the host galaxy, and we assume a circularly symmetric, singular isothermal mass distribution. For a mass distribution of this form, images are not stretched radially, but only tangentially, and the amplification factor $(\mu)$ of the amplification is identical to the factor by which the tangential component of the host galaxy is stretched.

We define the tangential ellipticity of a galaxy image as the quantity

$$
\epsilon_{T}=\frac{M_{T T}-M_{R R}}{M_{T T}+M_{R R}}=\frac{1-(b / a)^{2}}{1+(b / a)^{2}} \cos (2 \theta),
$$

where $M_{T T}$ and $M_{R R}$ are the second-order moments of the light distribution in the tangential and radial directions, respectively, $b / a$ is the ratio of minor to major axis of the light distribution, and $\theta$ is the angle between the major axis of the host galaxy and the tangential direction, $0^{\circ} \leq \theta \leq 90^{\circ}$. The angle $\theta=0^{\circ}$ if the source is tangentially oriented, and $\theta=90^{\circ}$ if the image is radially oriented. We use a suffix $I$ when referring to intrinsic quantities, without the distortion caused by lensing, and $M$ when referring to measured quantities.

In the presence of amplification $\mu>1$, the source is stretched tangentially by a factor $\mu$, while its radial size remains unchanged. The measured ellipticity $\epsilon_{T, M}$ is then related to the intrinsic ellipticity $\epsilon_{T, I}$ as

$$
\epsilon_{T, M}=\frac{\mu^{2}\left(1+\epsilon_{T, I}\right)-\left(1-\epsilon_{T, I}\right)}{\left(1+\epsilon_{T, I}\right)+\mu^{2}\left(1-\epsilon_{T, I}\right)} .
$$

The observed tangential ellipticity $\epsilon_{T, o}$ of the host galaxy can be estimated from its axis ratio $b / a=0.851$ and position angle of the major axis, $139^{\circ} .6$ east of north (M. Dickinson 2001, private communication). Since the lens is at a position angle of 
$11.3 \pm 0.7$ from the source, the major axis is 38.3 from the tangential direction, and the observed tangential ellipticity $\epsilon_{T, o}=0.037$ (i.e., quite small; see Fig. 1).

To determine how often the measured ellipticity $\epsilon_{T, M}$ of a galaxy like the host galaxy will be as small as the observed ellipticity $\epsilon_{T, o}$, we need to construct an appropriate distribution of the two relevant intrinsic image parameters, the intrinsic axis ratio $(b / a)_{I}$, and the intrinsic orientation angle $\theta_{I}$. Since there should be no physical interaction between lens and host galaxy, the orientation $\theta_{I}$ is distributed randomly and uniformly in the allowed range $0^{\circ} \leq \theta_{I} \leq 90^{\circ}$. We draw the axis ratio $(b / a)_{I}$ from the distribution observed by Lambas, Maddox, \& Loveday (1992) for galaxies classified as ellipticals in the APM survey. Although this distribution is obtained for low-redshift objects, we expect it to be representative of the high-redshift host galaxy because the latter is observed in the NIR (rest-frame optical) and does not appear significantly perturbed.

Figure 15 shows a subset $\left(10^{4}\right.$ of the $10^{6}$ samples) of randomly selected combinations of the intrinsic axis ratio and orientation angle of simulated host galaxies. For several different hypothetical values of the $\mathrm{SN}$ amplification, a curve is shown indicating the combinations of the intrinsic axis ratio and orientation angle resulting in the observed tangential eccentricity of the SN host. Intrinsic values above each curve correspond to galaxies for which the elongation and orientation are such as to produce a tangential ellipticity smaller than the observed value of 0.037 . Since many ellipticals are intrinsically nearly round $(b / a>0.7)$, for large assumed amplifications the intrinsic position angle must be very close to radial $\left(\theta_{I}>70^{\circ}\right)$ if the observed ellipticity is to be as small as observed. Each curve is labeled with the assumed amplification (in magnitudes) of the SN and the fraction of randomly selected galaxies that fall above it, i.e., those for which the observed tangential ellipticity would be 0.037 or smaller.

The small degree of observed tangential ellipticity of the SN host provides a useful constraint for the maximum likely amplification of the SN. (This determination is independent of the estimate in $\S 4.2$ of the expected amount of net amplification of the SN by both stochastic and specific lensing, $\sim 0.2$ mag.) For example, if we assumed that the SN was amplified by 0.6 mag by the foreground lens, we would conclude that we were relatively lucky (i.e., a $6 \%$ chance) to see such a small apparent tangential ellipticity for the host (a result which can occur for an E6 or E7 host galaxy intrinsically aligned within $20^{\circ}$ of the radial orientation). The chance of seeing such a small apparent tangential ellipticity for an amplification that moves the SN from the dust-filled universe model to its observed brightness $(\sim 1.1 \mathrm{mag})$ is less than $1 \%$.

Interestingly, the calculations above have predictive power for the other close lens candidate, located 5".3 from the host and also at $z=0.56$. This galaxy lies only $20^{\circ}$ from the radial axis joining the host and the closest lens. This lens would distort the host galaxy in a direction very similar to the one resulting from the closest lens.

Aguirre, A. 1999a, ApJ, 512, L19 $1999 \mathrm{~b}, \mathrm{ApJ}, 525,583$

Alard, C. 2000, A\&AS, 144, 363

Aldering, G., et al. 1998, IAU Circ. 7046

Balbi, A., et al. 2000, ApJ, 545, L1

Banerjee, S. K., Narlikar, J. V., Wickramasinghe, N. C., Hoyle, F., \& Burbidge, G. 2000, AJ, 119, 2583

Barbon, R., Ciatti, F., \& Rosino, L. 1979, A\&A, 72, 287

Bartunov, O. S., Tsvetkov, D. Yu., \& Filimonova, I. V. 1994, PASP, 106, 1276

Behnke, D., et al. 2001, preprint (gr-qc/0102039)

Bertin, E., \& Arnouts, S. 1996, A\&AS, 117, 393

Bruzual, A. G., \& Charlot, S. 1993, ApJ, 405, 538

Budavári, T., Szalay, A. S., Connolly, A. J., Csabai, I., \& Dickinson, M. 2000, AJ, 120, 1588

Caldwell, R. R., Davé, R., \& Steinhardt, P. J. 1998, Ap\&SS, 261, 303

Cappellaro, E., Evans, R., \& Turatto, M. 1999, A\&A, 351, 459

Cappellaro, E., Turatto, M., \& Fernley, J. 1995, IUE-ULDA Access Guide No. 6: Supernovae (Noordwijk: ESA)

Cappellaro, E., et al. 1997, A\&A, 322, 431

Cardelli, J. A., Clayton, G. C., \& Mathis, J. S. 1989, ApJ, 345, 245

Clocchiatti, A., et al. 2000, ApJ, 529, 661

Cohen, J. G., et al. 2000, ApJ, 538, 29

Coil, A., et al. 2000, ApJ, 544, L111

Coleman, G. D., Wu, C.-C., \& Weedman, D. W. 1980, ApJS, 43, 393 (CWW)

Cox, C., Ritchie, C., Bergeron, E., MacKenty, J., \& Noll, K. 1997, NICMOS STScI Instrument Science Report 97-07 (Baltimore: STScI)

de Bernardis, P., et al. 2000, Nature, 404,955

Dickinson, M. 1999, in After the Dark Ages: When Galaxies Were Young (the Universe at $2<z<5$ ), ed. S. Holt \& E. Smith (New York: AIP), 122

Drell, P. S., Loredo, T. J., \& Wasserman, I. 2000, ApJ, 530, 593

Ferguson, H. C., Dickinson, M., \& Williams, R. 2000, ARA\&A, 38, 667

Fernandez-Soto, A., Lanzetta, K. M., \& Yahil, A. 1999, ApJ, 513, 34

Filippenko, A. V. 1997, ARA\&A, 35, 309 2000, in Cosmic Explosions, ed. S. S. Holt \& W. W. Zhang (New York: AIP), 123

Filippenko, A. V., \& Riess, A. G. 2000, in Particle Physics and Cosmology: Second Tropical Workshop, ed. J. F. Nieves (New York: AIP), 227

Filippenko, A. V., et al. 1992a, ApJ, 384, L15 1992b, AJ, 104, 1543

Fruchter, A. S., \& Hook, R. N. 1997, Proc. SPIE, 3164, 120

Galama, T. J., et al. 1998, Nature, 395, 670

Germany, L. M., Reiss, D. J., Sadler, E. M., \& Schmidt, B. P. 2000, ApJ, 533,320

\section{REFERENCES}

Gilliland, R. L., Nugent, P. E., \& Phillips, M. M. 1999, ApJ, 521, 30 (GNP99)

Gilliland, R. L., \& Phillips, M. M. 1998, IAU Circ. 6810

Hamuy, M., et al. 1996, AJ, 112, 2408

.2000, AJ, 120, 1479

Höflich, P., \& Khokhlov, A. 1996, ApJ, 457, 500

Höflich, P., Wheeler, J. C., \& Thielemann, F. K. 1998, ApJ, 495, 617

Holz, D. E. 1998, ApJ, 506, L1

Howell, D. A. 2000, Ph.D. thesis, Univ. Oklahoma

Iwamoto, K., et al. 1998, Nature, 395, 672

Jaffe, A. et al. 2001, Phys. Rev. Lett., 86, 3475

Kennicutt, R. C. 1998, ARA\&A, 36, 189

Kim, A., Goobar, A., \& Perlmutter, S. 1996, PASP, 108, 190

Kirshner, R., et al. 1993, ApJ, 415, 589

Kobayashi, C., Tsujimoto, T., \& Nomoto, K. 2000, ApJ, 539, 26

Lambas, D. G., Maddox, S. J., \& Loveday, J. 1992, MNRAS, 258, 404

Leibundgut, B. 1988, Ph.D. thesis, Univ. of Basel

Leibundgut, B., et al. 1993, AJ, 105, 301

Lewis, G. F., \& Ibata, R. A. 2001, MNRAS, 324, L25

Li, W., Filippenko, A. V., \& Riess, A. G. 2001, ApJ, 546, 719

Li, W. D., et al. 1999, AJ, 117, 2709

Livio, M. 2000, in Type Ia Supernovae: Theory and Cosmology, ed. J. C.

Niemeyer \& J. W. Truran (Cambridge: Cambridge Univ. Press), 33

McLean, I., et al. 1998, Proc. SPIE, 3354, 566

Metcalf, R. B., \& Silk, J. 1999, ApJ, 519, L1

Modjaz, M., Li, W., Filippenko, A. V., King, J. Y., Leonard, D. C., Matheson, T., Treffers, R. R., \& Riess, A. G. 2001, PASP, 113, 308

Nomoto, K., Umeda, H., Hachisu, I., Kato, M., Kobayashi, C., \& Tsujimoto, T. 2000, in Type Ia Supernovae: Theory and Cosmology, ed. J. C. Niemeyer \& J. W. Truran (Cambridge: Cambridge Univ. Press), 63

Nugent, P. 2000, in Particle Physics and Cosmology: Second Tropical Workshop, ed. J. F. Nieves (New York: AIP), 263

Oke, J. B., \& Gunn, J. E. 1983, ApJ, 266, 713

Oke, J. B., et al. 1995, PASP, 107, 375

Pain, R., et al. 1996, ApJ, 473, 356

Peebles, P. J. E., \& Ratra, B. 1988, ApJ, 325, L17

Perlmutter, S., et al. 1995, ApJ, 440, L41

$$
\text { 1997, ApJ, 483, } 565
$$

1999, ApJ, 517,565

Pettini, M., Shapley, A. E., Steidel, C. C., Cuby, J.-G., Dickinson, M., Moorwood, A. F. M., Adelberger, K. L., \& Giavalisco, M. 2001, ApJ, 554, 981

Phillips, M. M. 1993, ApJ, 413, L105

Phillips, M. M., Lira, P., Suntzeff, N. B., Schommer, R. A., Hamuy, M., \&

Maza, J. 1999, AJ, 118, 1766

Phillips, M. M., et al. 1992, AJ, 103, 1632 
Pinto, P. A., \& Eastman, R. G. 2000, ApJ, 530, 744

Press, W. H., Teukolsky, S. A., Vetterling, W. T., \& Flannery, B. P. 1992 Numerical Recipes in FORTRAN (2d ed.; Cambridge: Cambridge Univ. Press)

Rana, N. C. 1979, Ap\&SS, 66, 173

. 1980, Ap\&SS, 71, 123

Riess, A. G. 2000, PASP, 112,1284

Riess, A. G., Press, W. H., \& Kirshner, R. P. 1996, ApJ, 473, 88

Riess, A. G., et al. 1997, AJ, 114, 722 1998, AJ, 116, 1009 2000, ApJ, 536, 62

Ruiz-Lapuente, P., \& Canal, R. 1998, ApJ, 497, L57

Saha, A., et al. 2001, ApJ, 551, 973

Sandage, A., \& Hardy, E. 1973, ApJ, 183, 743

Sawicki, M., Lin H., \& Yee, H. 1997, AJ, 113, 1

Schlegel, E. M. 1990, MNRAS, 244, 269

Schmidt, B. P., et al. 1994, ApJ, 432, 42

1998, ApJ, 507, 46

Shanks, T. Allen, P. D., Hoyle, F. \& Tanvir, N. R. 2001, in New Cosmological Data and the Value of the Fundamental Parameters, ed. A. N. Lasenby \& A. Wilkinson (San Francisco: ASP), in press (astro-ph/ 0102450)
Spinrad, H., Dey, A., Stern, D., Dunlop, J., Peacock, J., Jimenez, R., \& Windhorst, R. 1997, ApJ, 484, 581

Storrs, A., Hook, R., Stiavelli, M., Hanley, C., \& Freudling, W. 1999, NICMOS STScI Instrument Science Report 99-05 (Baltimore: STScI)

Sullivan, M., Ellis, R., Nugent, P., Smail, I., \& Madau, P. 2000, MNRAS, 319,549

Thompson, R. I., Storrie-Lombardi, L. J.,Weymann, R. J., Rieke, M. J., Schneider, G., Stobie, E., \& Lytle, D. 1999, AJ, 117, 17

Tonry, J., et al. 1999, IAU Circ. 7312

Turatto, M., et al. 2000, ApJ, 534, L57

Umeda, H., Nomoto, K., Kobayashi, C., Hachisu, I., \& Kato, M. 1999a, ApJ, 522, L43

Umeda, H., Nomoto, K., Yamaoka, H., \& Wanajo, S. 1999b, ApJ, 513, 861

Williams, R. E., et al. 1996, AJ, 112, 1335

Woosley, S. E., Eastman, R. G., \& Schmidt, B. P. 1999, ApJ, 516, 788

Yungelson, L. R., \& Livio, M. 2000, ApJ, 528, 108 